\title{
Quantitative Examinations of Internal Representations for Arm Trajectory Planning: Minimum Commanded Torque Change Model
}

\author{
ERI NAKANO, ${ }^{1,2}$ HIROSHI IMAMIZU, ${ }^{3}$ RIEKO OSU, ${ }^{3}$ YOJI UNO, ${ }^{4}$ HIROAKI GOMI, ${ }^{5}$ TOSHINORI YOSHIOKA, ${ }^{3}$ \\ AND MITSUO KAWATO ${ }^{1,3}$ \\ ${ }^{1}$ ATR Human Information Processing Research Laboratories, Kyoto 619-0288; ${ }^{2}$ Graduate School of Science and Technology, \\ Kobe University, Hyogo 657-0013; ${ }^{3}$ Kawato Dynamic Brain Project, ERATO, Japan Science and Technology Corporation, \\ Kyoto 619-0288; ${ }^{4}$ Department of Information and Computer Sciences, Toyohashi University of Technology, Aichi 441-8540; \\ and ${ }^{5}$ NTT Communication Science Laboratories, Nippon Telegraph and Telephone Corporation, Kanagawa 243-0198, Japan
}

\begin{abstract}
Nakano, Eri, Hiroshi Imamizu, Rieko Osu, Yoji Uno, Hiroaki Gomi, Toshinori Yoshioka, and Mitsuo Kawato. Quantitative examinations of internal representations for arm trajectory planning: minimum commanded torque change model. J. Neurophysiol. 81: 2140-2155, 1999. A number of invariant features of multijoint planar reaching movements have been observed in measured hand trajectories. These features include roughly straight hand paths and bell-shaped speed profiles where the trajectory curvatures between transverse and radial movements have been found to be different. For quantitative and statistical investigations, we obtained a large amount of trajectory data within a wide range of the workspace in the horizontal and sagittal planes (400 trajectories for each subject). A pair of movements within the horizontal and sagittal planes was set to be equivalent in the elbow and shoulder flexion/extension. The trajectory curvatures of the corresponding pair in these planes were almost the same. Moreover, these curvatures can be accurately reproduced with a linear regression from the summation of rotations in the elbow and shoulder joints. This means that trajectory curvatures systematically depend on the movement location and direction represented in the intrinsic body coordinates. We then examined the following four candidates as planning spaces and the four corresponding computational models for trajectory planning. The candidates were as follows: the minimum hand jerk model in an extrinsic-kinematic space, the minimum angle jerk model in an intrinsic-kinematic space, the minimum torque change model in an intrinsic-dynamic-mechanical space, and the minimum commanded torque change model in an intrinsic-dynamic-neural space. The minimum commanded torque change model, which is proposed here as a computable version of the minimum motor command change model, reproduced actual trajectories best for curvature, position, velocity, acceleration, and torque. The model's prediction that the longer the duration of the movement the larger the trajectory curvature was also confirmed. Movements passing through via-points in the horizontal plane were also measured, and they converged to those predicted by the minimum commanded torque change model with training. Our results indicated that the brain may plan, and learn to plan, the optimal trajectory in the intrinsic coordinates considering arm and muscle dynamics and using representations for motor commands controlling muscle tensions.
\end{abstract}

\section{N T R O D U C T I O N}

Hand trajectories measured for planar reaching movements are known to have common invariant spatiotemporal features, namely, a roughly straight hand path, a bell-shaped tangential velocity profile (Abend et al. 1982; Flash and Hogan 1985;

The costs of publication of this article were defrayed in part by the payment of page charges. The article must therefore be hereby marked "advertisement" $n$ accordance with 18 U.S.C. Section 1734 solely to indicate this fact.
Kelso et al. 1979; Morasso 1981; Uno et al. 1989a), and smooth acceleration (Koike and Kawato 1995). These invariant features can be observed in rapidly executed movements without on-line correction. The hand trajectory seems to be planned for the execution of such a feed-forward controlled movement. The experiments by Bizzi et al. (1984) suggest that deafferented monkeys can reach a target with their hands by feedforward control alone, and the whole trajectory from the initial position to the final position is preplanned.

Three "indeterminacy problems" are involved in planning and executing reaching tasks with a visually guided arm (Kawato 1992). There are an infinite number of spatiotemporally possible routes leading to the target, but it is necessary to select a final unique trajectory (trajectory determination problem). Even if the hand position is determined in the extrinsic coordinates, the joint angles or the muscle lengths cannot be uniquely determined because of redundant degrees of freedom (a problem of coordinates transformation). When a desired trajectory is determined in the joint angle coordinate, actual torques around the joints can be calculated by an inverse dynamics equation. However, there are also an infinite number of possible combinations of the agonist and antagonist muscle tensions that can generate the same torques. The degrees of freedom of $\alpha$-motoneurons, which innervate each muscle, are higher than those of the muscles, and cortical motor neurons may have higher degrees of freedom than $\alpha$-motoneurons. Even if the time profiles of muscle tensions are specified, the firing rates of the cortex or spinal cord neurons cannot be uniquely determined (a problem of motor command generation). Regardless of these indeterminacies, the actual hand trajectories show common invariant characteristics, and electromyographic (EMG) signals appear in typical triphasic patterns. These observations suggest that the brain solves these ill-posed problems based on some principles (for further detail of these problems, see Kawato 1996).

Many approaches have been proposed to explain how the brain resolves such problems (Bernstein 1967; Bizzi et al. 1984; Saltzman and Kelso 1987). Optimization models have been proposed for single joint movements (Nelson 1983) and for multijoint movements (Dornay et al. 1996; Flash and Hogan 1985; Kawato 1992, 1996; Uno et al. 1989a,b). These models are objectively and experimentally examinable because of their quantitative predictions. It has already been confirmed that most of the criteria proposed for single joint movements 
are unable to reproduce the smoothness of the velocity or acceleration in multijoint movements (Kawato 1996). In addition, the combination of the virtual trajectory and minimum jerk models proposed by Flash (1987) is also a quantitatively examinable model. The claims or advantages of this model are high arm stiffness, invariance of virtual trajectory, and simple desired trajectory (straight virtual trajectory). However, the arm stiffness measured during movement is not high (Gomi and Kawato 1996, 1997). ${ }^{1}$ The invariance of virtual trajectory does not hold well because not only the amplitude but also the temporal patterns of EMG signals are different between straight and natural movements (Osu et al. 1997). A quite different trajectory from the actual one is predicted with actually measured low stiffness and a straight virtual trajectory (Katayama and Kawato 1993). Due to these reasons, this model does not seem to be an attractive candidate for now.

Because most targets for movements are provided in external visual coordinates and the achieved trajectory is roughly straight, it seems natural at first sight that the necessary constraints to solve the indeterminacy problem are in the extrinsic coordinates (Flash and Hogan 1985). However, the possibility that trajectories are planned in intrinsic joint torque or motor command space has been pointed out (Kawato 1992; Uno et al. 1989a). Different spaces where optimization principles are applied predict different trajectory properties. Hence, in this study, we discuss the problem of the planning space by experimentally investigating the properties of executed trajectories. As a candidate of the coordinates frame for trajectory planning, we first considered the extrinsic coordinates represented by factors such as the position within the task space, and the intrinsic coordinates based on inherent expressions in the motor system such as the joint angle, muscle length, muscle tension, torque, and motor command. Second, following on the work of Osu et al. (1997), we classified spaces that either solely rely on kinematic aspects, e.g., the hand position, joint angle, and muscle length, or on both kinematic and dynamic aspects, e.g., torque and muscle tension. Finally, the dynamic parameters are divided into mechanical variables, e.g., torque and muscle tension, and neural representations that depend on nervous system processing, e.g., the motor command controlling muscle tension. These three methods of classification allow us to consider the following four plausible candidates as planning spaces: an extrinsic-kinematic space (e.g., the Cartesian coordinates of the hand position, hand movement direction, or the polar coordinates), an intrinsic-kinematic space (e.g., joint angle or muscle length), an intrinsic-dynamic-mechanical space (e.g., torque or muscle tension), and an intrinsic-dynamic-neural space (e.g., the motor command control-

\footnotetext{
${ }^{1}$ Gribble et al. (1998) presented a different interpretation of data of Gomi and Kawato (1996) using a complicated nonlinear muscle model. Although their muscle model is complicated and looks biologically plausible, it is not a quantitatively validated model. For example, their model is an overdamped system that is against the previous observations that the musculoskeletal system is underdamped. Another trivial mistake of their approach is that they simulated free movements while using parameter values derived from constrained movements. Subjects in Gomi and Kawato (1996) generated $\sim 2.5$ times larger torques compared with free movements because of the manipulandum load. Thus the observed stiffness in the experiment is expected several times larger than in movements without any load. If the same load with the experiments were applied in the simulation of Gribble et al. (1998), our experimental results (movements and stiffness) may not be reproduced by constant change of equilibrium position.
}

ling muscle tensions, the firing rates of motor neurons, or Purkinje cells in the cerebellum).

Neural recording data consistent with the four different planning spaces have been obtained. Examples are Georgopoulos et al. $(1982,1986)$ for an extrinsic-kinematic space, Lacquaniti et al. (1995) for an intrinsic-kinematic space, Scott and Kalaska (1995, 1997), Sergio and Kalaska (1998) for an intrinsic-dynamic-mechanical space, and Keller (1973), Shidara et al. (1993), and Gomi et al. (1998) for an intrinsic-dynamicneural space. Especially, for eye movements, it has been reported that firing frequencies of motor neurons (Fuchs et al. 1988; Keller 1973) and cerebellar Purkinje cells (Gomi et al. 1998) represent dynamic motor commands specifying necessary muscle forces and torques. Sergio and Kalaska (1998) showed in arm control that each firing pattern of the primary motor cortex neurons obtained in different movement tasks is similar to the corresponding temporal profile of force necessary in each task.

Recent studies have investigated performance changes or adaptation processes in artificially altered environments to objectively discuss the trajectory planning space. If a hand trajectory is planned in a kinematic space, it will be changed under a kinematic transformation in the visual space, but it will not be affected by any altered dynamics, e.g., the force field where the viscosity was altered. However, the dynamic model makes the opposite prediction. Results supporting kinematic planning have been reported by both Wolpert et al. (1995), and Flanagan and Rao (1995) using kinematic transformation, and by Flash and Gurevich (1991), Shadmehr and Mussa-Ivaldi (1994), Lackner and Dizio (1994), and Conditt et al. (1997) using dynamic transformation. On the contrary, the results of Uno et al. (1989a), Uno et al. (1995), Osu et al. (1997), Gomi and Gottlieb (1997), and Uno, Imamizu, and Kawato (unpublished observations) support dynamic planning. The differences in these results can be ascribed to whether or not the internal model for the alternation has been sufficiently learned or the transformation is strong enough to change the optimal hand trajectory (Kawato 1996). This controversial problem will continue, because the results depend on the settings of delicate task conditions, the number of learning trials, and the instructions given in the transformation experiments. In this paper, we adopted the tasks under ordinary space without using the transformation to discuss trajectory planning spaces.

One way to investigate the space in which trajectories are planned is to compare actual trajectories with trajectories predicted by the optimization criterion defined in each space. We used the minimum hand jerk (Flash and Hogan 1985), minimum angle jerk, minimum torque change (Uno et al. 1989a), and minimum commanded torque change models, whose objective functions are defined in the above spaces, respectively. In the next section, we describe four optimal trajectory formation models and specifically propose the minimum commanded torque change model. The diagram in Fig. 1 shows the spaces for trajectory planning and the corresponding models. In a comparison of actual trajectories with predicted trajectories, the fundamental assumption is that actual trajectories are close to planned trajectories. This assumption is confirmed by Osu et al. (1997), and by partially referring to Katayama and Kawato (1993) and Gomi and Kawato (1996).

Utilization of data obtained in limited locations makes it possible for the conclusion to be dominated by the selected 


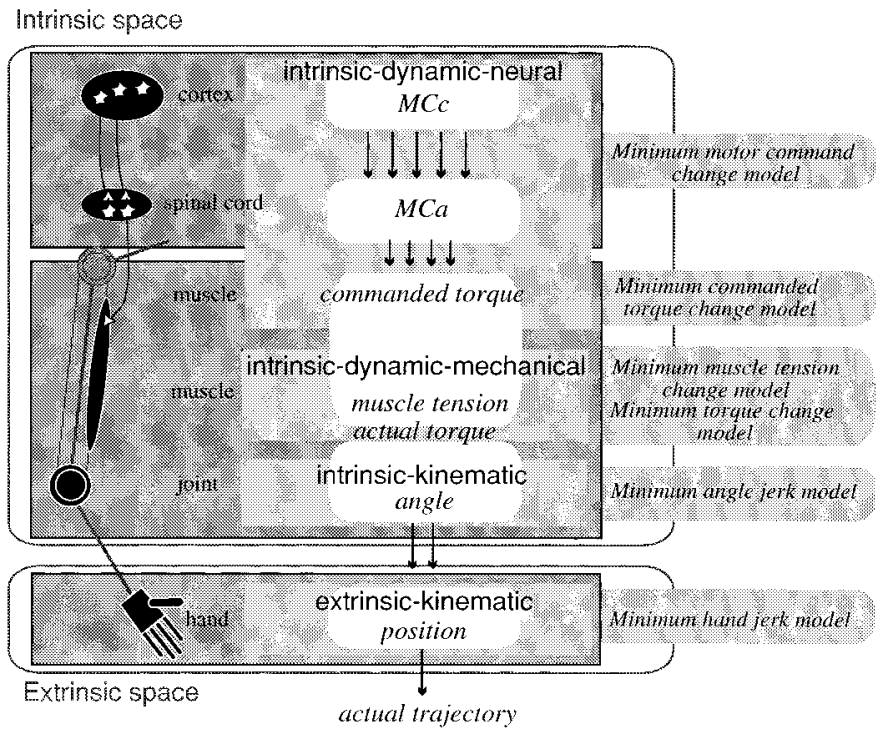

FIG. 1. Conceptual schema of trajectory planning spaces. Motor cortex conveys motor commands MCc to $\alpha$-motoneuron of the spinal cord, and the motor command $\mathrm{MC} \alpha$ derived from the $\alpha$-motoneuron activates muscles it innervates. Muscle activation is controlled by these impulses, muscle tensions then arise, and finally actuated torques are generated to realize trajectory.

trajectories. There is also the possibility that the differences between model predictions are small for some specific movements. Hence generalized studies should utilize a large number of movements executed at many possible locations within the workspace.

In the first experiment, a large amount of data on point-topoint movements was obtained in the horizontal plane at shoulder level and in the sagittal plane passing through the shoulder. First, the relationship of trajectory curvatures between movements executed in the horizontal plane and those executed in the sagittal plane was investigated. Second, the trajectory curvatures were modeled from the locations and directions where the movements were accomplished. Third, we used the data to compute optimal trajectories to explore the space where the trajectories were planned.

In the second experiment, we examined the influence of the duration of movements on hand trajectories because dynamic planning models, but not kinematic planning models, predict changes in the trajectory with movement duration.

The subjects were requested to train for via-point movements in the third experiment. We compared the characteristics of measured trajectories and the characteristics of optimal trajectories for each model. Note that trajectory planning models have previously been examined for via-point movements (Flash and Hogan 1985; Okadome and Honda 1992; Uno et al. 1989a). However, studies have yet to examine each model by observing changes in trajectory properties due to training.

\section{Minimum commanded torque change model}

The minimum hand jerk model (Flash and Hogan 1985), defined in an extrinsic-kinematic space, is an attractive candidate as a trajectory planning model for humans. Here, the jerk is defined by differentiating the hand position $(x, y)$ three times by time $t$ in Cartesian coordinates. In this model, the objective function

$$
C_{\mathrm{J}}=1 / 2 \int_{0}^{t_{\mathrm{f}}}\left[\left(\mathrm{d}^{3} x / \mathrm{d} t^{3}\right)^{2}+\left(\mathrm{d}^{3} y / \mathrm{d} t^{3}\right)^{2}\right] \mathrm{d} t
$$

is minimized, where $t_{\mathrm{f}}$ is the movement duration. This model always predicts straight paths regardless of the influence of arm dynamics, arm posture, external forces, and movement duration.

Soechting and Lacquaniti (1981) discussed trajectory planning in a kinematic joint space based on the observation that the shoulder and elbow move while maintaining a linear relationship between the joint angles near the edge of the workspace. Rosenbaum et al. (1995) have discussed the coordinated movements of arm and trunk using optimization criteria defined in the joint space. This model is named here the minimum angle jerk model as one possible exemplification of the optimization theory. This model always predicts straight paths in the joint space. The criterion function to be minimized is expressed as

$$
C_{\mathrm{AJ}}=1 / 2 \int_{0}^{t_{\mathrm{f}}} \sum_{i=1}^{n}\left(\mathrm{~d}^{3} \theta_{i} / \mathrm{d} t^{3}\right)^{2} \mathrm{~d} t
$$

where $\theta_{i}$ is the $i$ th joint out of $n$ joints. Because a straight trajectory in the joint space was thought to be much more curved than the actual trajectory in the extrinsic hand space, this class of model has been considered to be inappropriate (Hollerbach 1990; Osu et al. 1997). However, we will quantitatively demonstrate that this model can predict the actual trajectory curvature better than the minimum hand jerk model.

Trajectory planning in an intrinsic-dynamic space is another candidate capable of accounting for actual data. The minimum torque change model (Uno et al. 1989a) is classified as "intrinsic-dynamic-mechanical." This model was able to reproduce the properties of hand trajectory influenced by arm dynamics, arm posture, external forces, and movement duration (Uno et al. 1989a; Uno and Kawato 1996). However, as is detailed later, it has been pointed out that the viscous values should be set to zero in the literal minimum torque change model (Flash 1990), and this literal model is discriminated from our new one, the minimum commanded torque change model.

The formulas of the original minimum torque change model (Uno et al. 1989a) and the minimum commanded torque change model proposed here are the same and both include the viscous values. However, the original paper by Uno et al. (1989a) misnamed the model and used incorrect values for inertia and viscosity. The actual trajectories are not at all similar to the trajectories predicted by using our viscous values and an inertia value by Uno et al. (1989a) that is too large or those predicted by using our inertia value and the viscous values of Uno et al. (1989a) that are too small (but not zero). The combination of wrong parameters (large inertia and small viscous values) contingently leads the prediction of trajectories very similar to the actual ones. Therefore our minimum commanded torque change trajectory is at first sight similar to the original minimum torque change trajectory. However, our paper provided appropriate parameters, accurate interpretation, and proper designation to the original model.

The minimum motor command change model (Kawato 1992, 1996) has been proposed for trajectory planning in an 
intrinsic-dynamic-neural space. The bursting of motor neurons or the cerebellar Purkinje cells observed in rapid movements, e.g., saccadic eye movements or ocular following responses, apparently seems to go against the principle of smoothness. However, a bell-shaped velocity profile and smooth acceleration were observed even in saccades as well as in arm movements (Harris and Wolpert 1998). It has been demonstrated that the temporal profile of the firing frequency of motor neurons (Fuchs et al. 1988; Keller 1973) or the cerebellar Purkinje cells (Gomi et al. 1998; Shidara et al. 1993) changes according to such smooth temporal profiles of velocity and acceleration of eye movements. Gomi et al. (1998) reported that the firing patterns of cerebellar Purkinje cells were represented by a linear summation of position, velocity, and acceleration and smoothly changed over time correlating with the dynamic component of the necessary torque. Consequently, smoothness of central motor commands has already been observed in neuronal recording data.

It is reasonable to consider that the principle of smoothness should be applied in the motor command space because the degrees of freedom are higher at the CNS level as mentioned in the INTRODUCTION. Because the minimum motor command change model can conceptualize the signal at the $\alpha$-motoneuron or cortical motoneuron level, the indeterminacy can be constrained at each level. Although an attempt has been made to estimate the motor commands at the muscle level (Koike and Kawato 1995), it is extremely difficult to estimate the motor commands of the spinal cord or cortex by modeling the information processing from a central system to a peripheral system. A quantitative model, not a conceptual model, is needed to actually compute an optimal trajectory. Therefore we are the first to fully propose a minimum commanded torque change model that approximates the minimum motor command change model and has computability, while positively appreciating the assumption of nonzero viscosity by Uno et al. (1989a). In the literal minimum torque change model, only the link dynamics are regarded as the controlled object, whereas in the minimum commanded torque change model, both link dynamics and muscles are regarded as controlled objects (Fig. 2). We employ motor commands at the peripheral level, in other words, we use signals controlling muscle tensions to model a minimum commanded torque change criterion. In terms of indeterminacy, however, the minimum commanded torque change model solves problems at the same level, that is, the torque level as the minimum torque change model.

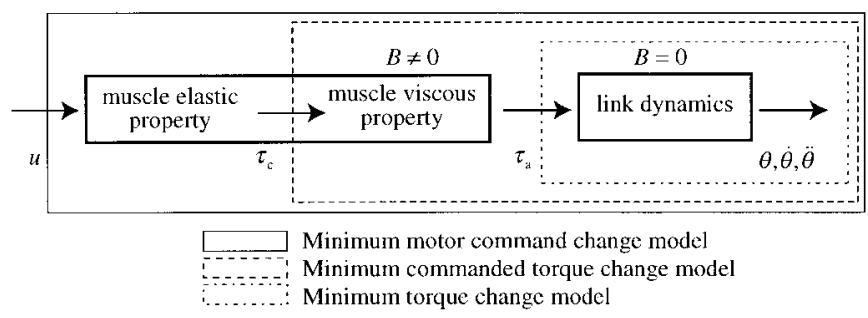

FIG. 2. Conceptual block diagram for intrinsic-dynamic models. Motor command $u$ is transmitted to muscle and commanded torque $\tau_{\mathrm{c}}$ is generated through muscle elastic property. $\tau_{\mathrm{c}}$ is dumped by muscle viscous property, and actual torque $\tau_{\mathrm{a}}$ is obtained. $u$ designates $\tau_{\mathrm{c}}$ capable of producing necessary $\tau_{\mathrm{a}}$ to complete a given movement considering torque deducted through viscous property. Minimum torque change model only regards link dynamics as controlled object $(-\cdot \cdot-)$, minimum commanded torque change model regards both link dynamics and muscles as controlled objects (-- ).
TABLE 1. Values of physical parameters of the arm

\begin{tabular}{|c|c|c|c|}
\hline \multirow[b]{2}{*}{ Parameters } & \multicolumn{3}{|c|}{ Subjects } \\
\hline & $M M$ & $Y S$ & $T T$ \\
\hline $\mathrm{M}_{1}, \mathrm{~kg}$ & 1.41 & 1.30 & 1.50 \\
\hline $\mathrm{M}_{2}, \mathrm{~kg}$ & 1.08 & 1.07 & 1.11 \\
\hline $\mathrm{L}_{1}, \mathrm{~m}$ & 0.285 & 0.265 & 0.345 \\
\hline $\mathrm{L}_{2}, \mathrm{~m}$ & 0.335 & 0.330 & 0.300 \\
\hline $\mathrm{S}_{1}, \mathrm{~m}$ & 0.11 & 0.10 & 0.11 \\
\hline $\mathrm{S}_{2}, \mathrm{~m}$ & 0.16 & 0.16 & 0.17 \\
\hline $\mathrm{I}_{1}, \mathrm{~kg} \cdot \mathrm{m}^{2}$ & 0.025 & 0.020 & 0.029 \\
\hline \multirow[t]{2}{*}{$\mathrm{I}_{2}, \mathrm{~kg} \cdot \mathrm{m}^{2}$} & 0.043 & 0.042 & 0.047 \\
\hline & \multicolumn{3}{|c|}{ Horizontal } \\
\hline Mean $\mathrm{B}_{11}, \mathrm{~kg} \cdot \mathrm{m}^{2} / \mathrm{s}$ & 0.72 & 0.71 & 0.74 \\
\hline Mean $\mathrm{B}_{22}, \mathrm{~kg} \cdot \mathrm{m}^{2} / \mathrm{s}$ & 0.81 & 0.81 & 0.82 \\
\hline Mean $\mathrm{B}_{12}, \mathrm{~kg} \cdot \mathrm{m}^{2} / \mathrm{s}$ & 0.08 & 0.08 & 0.10 \\
\hline \multirow[t]{2}{*}{ Mean $\mathrm{B}_{21}, \mathrm{~kg} \cdot \mathrm{m}^{2} / \mathrm{s}$} & 0.08 & 0.08 & 0.10 \\
\hline & \multicolumn{3}{|c|}{ Sagittal } \\
\hline Mean $\mathrm{B}_{11}, \mathrm{~kg} \cdot \mathrm{m}^{2} / \mathrm{s}$ & 0.87 & 0.85 & 0.90 \\
\hline Mean $\mathrm{B}_{22}, \mathrm{~kg} \cdot \mathrm{m}^{2} / \mathrm{s}$ & 1.00 & 0.99 & 1.00 \\
\hline Mean $\mathrm{B}_{12}, \mathrm{~kg} \cdot \mathrm{m}^{2} / \mathrm{s}$ & 0.27 & 0.26 & 0.27 \\
\hline Mean $B_{21}, \mathrm{~kg} \cdot \mathrm{m}^{2} / \mathrm{s}$ & 0.27 & 0.26 & 0.27 \\
\hline
\end{tabular}

In the minimum torque change model and minimum commanded torque change model, the objective function to be minimized is expressed by

$$
C_{\tau}=1 / 2 \int_{0}^{t_{f}} \sum_{i=1}^{n}\left(\mathrm{~d} \tau_{i} / \mathrm{d} t\right)^{2} \mathrm{~d} t
$$

where $\tau_{\mathrm{i}}$ is either the actual torque in the minimum torque change model, or the commanded torque in the minimum commanded torque change model, generated around the $i$ th joint out of $n$ joints. The shoulder is 1 , and the elbow is 2 . The actual torque with zero viscosity or commanded torque with nonzero viscosity is computed from the following dynamics equation of a two-link manipulator

$$
\begin{aligned}
\tau_{1}=\left[I_{1}\right. & \left.+I_{2}+2 M_{2} L_{1} S_{2} \cos \theta_{2}+M_{2}\left(L_{1}\right)^{2}\right] \ddot{\theta}_{1} \\
+ & \left(I_{2}+M_{2} L_{1} S_{2} \cos \theta_{2}\right) \ddot{\theta}_{2}-M_{2} L_{1} S_{2}\left(2 \dot{\theta}_{1}+\dot{\theta}_{2}\right) \dot{\theta}_{2} \sin \theta_{2} \\
+ & B_{11} \dot{\theta}_{1}+B_{12} \dot{\theta}_{2}+g\left[\left(M_{1} S_{1}+M_{2} L_{1}\right) \sin \theta_{1}+M_{1} S_{2} \sin \left(\theta_{1}+\theta_{2}\right)\right] \\
\tau_{2}=\left(I_{2}+M_{2} L_{1} S_{2} \cos \theta_{2}\right) \ddot{\theta}_{1} & +I_{2} \ddot{\theta}_{2}+M_{2} L_{1} S_{2}\left(\dot{\theta}_{1}\right)^{2} \sin \theta_{2} \\
& +B_{22} \dot{\theta}_{2}+B_{21} \dot{\theta}_{1}+g M_{2} S_{2} \sin \left(\theta_{1}+\theta_{2}\right)
\end{aligned}
$$

$I_{\mathrm{i}}, M_{\mathrm{i}}, L_{\mathrm{i}}, S_{\mathrm{i}}$, and $g$ represent inertia, mass, arm length, center of mass of link $i$, and acceleration due to gravity $\left(9.8\left[\mathrm{~m} / \mathrm{s}^{2}\right]\right)$. Links 1 and 2 correspond to the upper arm and the forearm. The values of $I_{\mathrm{i}}, M_{\mathrm{i}}$, and $S_{\mathrm{i}}$ are estimated from the measured link length $L_{\mathrm{i}}$ for each subject. ${ }^{2} B_{\mathrm{ij}}$ is the viscosity coefficient expressing the influence of the angular velocity of joint $j$ on the torque of joint $i$. Table 1 summarizes the physical parameters of the arm for each subject. Value $g$ is set to zero for experiments in the horizontal plane because a table supports the arm.

\footnotetext{
${ }^{2}$ The three-dimensional shape of a male's arm was measured by a Cyberware Laser Range Scanner. We calculated the arm as a homogeneous material with a specific gravity of 1.0 and computed its mass, center of mass, and moment of inertia from its volume. The arm parameters for each subject were calculated using the ratio of the arm length based on the measured data.
} 
In the sagittal plane, torques are computed by considering the force supporting the arm against the acceleration of gravity $g$.

Most of the viscosity measured around a joint is ascribed to a biochemical and mechanical reaction process within the muscle when it receives impulses and generates tension, which is not ascribed to a passive property of the joint (Akazawa 1994). Considering viscosity in calculating torque means that both link dynamics and muscles are regarded as controlled objects (Fig. 2). It is not appropriate to use the nonzero viscous value to calculate torques because the literal minimum torque change model takes the actual torque around the joint as an object for optimization (Flash 1990).

The commanded torque is calculated in consideration of the muscle viscosity and is intended to conceptually approximate the command of the $\alpha$-motoneuron MC $\alpha$ (see Fig. 1). The term commanded torque indicates the torques ascribed to a muscle elastic property, which reflects the motor command mainly controlling the rest-length of a muscle. In other words, the motor command controlling muscle tensions and consequently muscle torques designate this commanded torque. The commanded torque includes a component for compensating damping by muscle viscous properties (viscous torque), namely, the commanded torque is torque before being damped by such viscous properties (Fig. 2). To generate actual torque, the motor command controlling muscle tensions must compensate for the influence of muscle viscosity. By considering link dynamics as well as muscle properties, the commanded torque reflects a representation of a motor command more closely than the actual torque.

This conception is illustrated by the following expressions. The equations conceptually interpret a model approximation of the minimum motor command change model. We expressed $\tau$ in a vector form in Eqs. 2-5. The terms depending on position, velocity, and acceleration in Eq. 1 were rearranged using different symbols for concise explanation. The predicted trajectories were actually calculated by Eq. 1 but not by Eqs. 2-5. The minimum commanded torque change model does not model the cortical or spinal motor command itself. Here, we use the term motor command as the command already conveyed to the muscle and as that which controls tensions at the muscle level. That is to say, the influence of reflexes at the spinal or cortical level has been already involved in this motor command. Because there is no neural delay included in this final torque generating process, we did not consider any neural delay in the following equations.

Equation 2 defines the actual torque $\tau_{\mathrm{a}}$. The first and second terms in Eq. 1 correspond to the term $R$ in $E q$. 2. The third and sixth terms are expressed by the term $H$ in $E q$. 2 . We assume that there is no passive joint viscosity. The actual torque is determined as Eq. $3 a$ according to the Kelvin-Voight model (Özkaya and Norbin 1991). $R, H, K$, and $B_{\mathrm{m}}$ denote the inertia matrix, the centrifugal force and the Coriolis force, the stiffness matrix, and the muscle viscosity matrix, respectively. We defined the component ascribed to the muscle out of $B_{\mathrm{ij}}$ in $E q$. 1 as $B_{\mathrm{m}}$ in $E q .3 a$. $\theta$ and $\theta_{\mathrm{r}}$ represent the current position and the equilibrium position of the joint in the vector form. The elements of the matrix $K$ are coefficients of joint stiffness due to each muscle elasticity. This first elastic term in $E q .3 a$ is defined as the commanded torque $\tau_{\mathrm{c}}(E q .3 b)$. The negative designated $B_{\mathrm{m}}$ indicates that $\tau_{\mathrm{c}}$ is reduced by viscous force relating to the shortening velocity of the muscle. The com- manded torque can then be rewritten as Eq. 4, which explicitly shows that the commanded torque considers viscous force. The actual torque does not consider it (Eq. 2)

$$
\begin{gathered}
\tau_{\mathrm{a}}(\theta, \dot{\theta}, u)=R(\theta) \ddot{\theta}+H(\theta, \dot{\theta}) \\
\begin{aligned}
\tau_{\mathrm{a}}(\theta, \dot{\theta}, u) & =K(u)\left[\theta-\theta_{\mathrm{r}}(u)\right]-B_{\mathrm{m}}(u) \dot{\theta} \\
& \equiv \tau_{\mathrm{c}}(\theta, u)-B_{\mathrm{m}}(u) \dot{\theta} \\
\tau_{\mathrm{c}}(\theta, u)= & R(\theta) \ddot{\theta}+H(\theta, \dot{\theta})+B_{\mathrm{m}}(u) \dot{\theta}
\end{aligned}
\end{gathered}
$$

The actual torque can be further expressed as Eq. $5 a$ assuming that $K(u), \theta_{\mathrm{r}}(u)$, and $B_{\mathrm{m}}(u)$ have the linear dependence with respect to motor command $u$ (Katayama and Kawato 1993)

$$
\begin{aligned}
\tau_{\mathrm{a}}(\theta, \dot{\theta}, u) & =\left(k_{0}+k_{1} u\right)\left[\theta-\left(\theta_{0}-r u\right)\right]-\left(b_{0}^{\mathrm{m}}+b_{1}^{\mathrm{m}} u\right) \dot{\theta} \\
& =k_{0}\left(\theta-\theta_{0}\right)+\left[\left(\theta-\theta_{0}\right) k_{1}+r k_{0}\right] u+k_{1} r u^{2} \\
& \approx p+q u-B_{\mathrm{m}}(u) \dot{\theta}
\end{aligned}
$$

In this formula, $k_{1}$ and $b_{1}^{\mathrm{m}}$ represent the coefficients of $u$ that express elasticity and viscosity. The intrinsic elasticity and viscosity independent of the motor command in the musculoskeletal system are denoted by $k_{0}$ and $b_{0}^{\mathrm{m}}$. $\theta_{0}$ expresses the equilibrium point when the motor command is zero. $r$ is a coefficient of $u$ determining the equilibrium point. The term $p$ in $E q .5 c$ corresponds to the first term of Eq. $5 b$. The second and third terms of $E q .5 b$ are approximated to the term $q u$, which is dependent on the motor command assuming that change in $\theta$ is slower than change in $u$, and the higher order term of $u$ is negligible. According to the minimum commanded torque change model, the change over time of the second term in $E q .5 c$, namely the motor command change, is thought to be approximately minimized.

The coefficient of viscosity is known to vary with joint angle, angular velocity, or stiffness during postural control and movement (Bennet 1993; Bennet et al. 1992; Gomi and Osu 1998). No study has ever reported the viscous value during multijoint movements. In our study, we use the following formula, which was estimated from the actual torque and viscosity during static force control (Gomi and Osu 1998), to acquire viscous values of diagonal components $\left(B_{11}, B_{22}\right)$ and off-diagonal components $\left(B_{21}, B_{12}\right)$ for each trajectory. Here, for simplicity, mean absolute torques (shoulder: $\tau_{1}^{\mathrm{ma}}$, elbow: $\tau_{2}^{\mathrm{ma}}$ ) during movement are used as the actual torques

$\left[B_{11}, B_{12}\left(=B_{21}\right), B_{22}\right]=\left[0.63+0.095 \tau_{1}^{\mathrm{ma}}, 0.175+0.0375 \tau_{2}^{\mathrm{ma}}, 0.76\right.$

$$
\left.+0.185 \tau_{2}^{\mathrm{ma}}\right] \quad \mathrm{Nm} /(\mathrm{rad} / \mathrm{s})
$$

In this study, we compute the literal minimum torque change trajectories with zero viscosity, and the minimum commanded torque change trajectories with viscous values estimated by using $E q$. 6. We note that the minimum torque change model we test in this paper uses appropriate inertial and viscous parameters and minimizes torque change assuming zero viscosity. No parameter fitting was performed for any model.

\section{METHODS}

\section{Experimental setup}

POINT-TO-POINT MOVEMENTS. In the first experiment, the subjects were three right-handed males, 22-26 yr old (MM, YS, and TT). They sat 


\section{A}
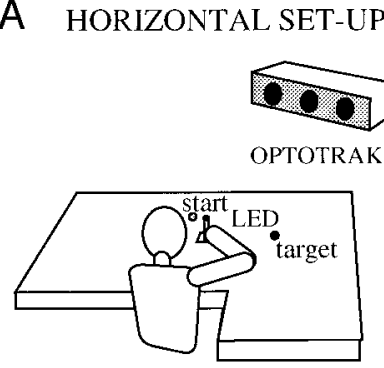

C

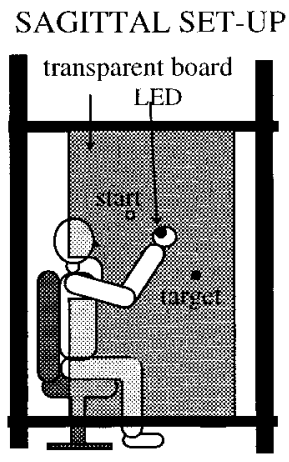

E VIA-POINT SET-UP

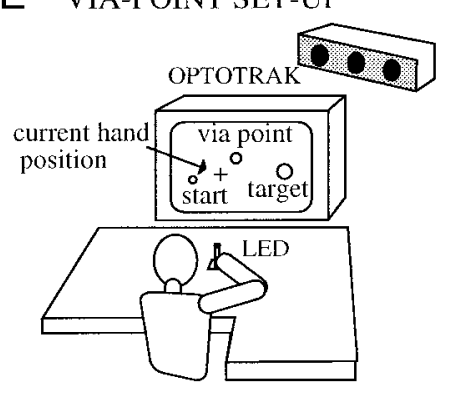

\section{B HORIZONTAL WORKSPACE}

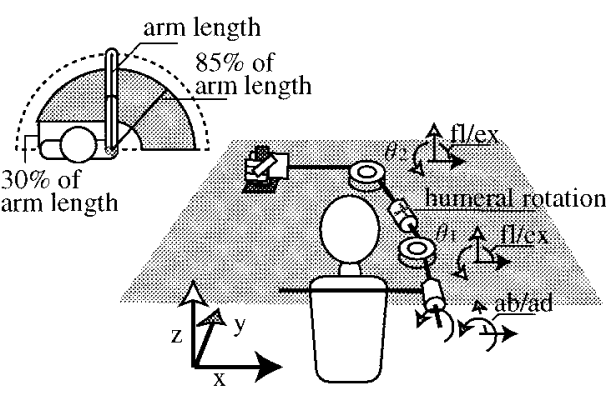

D SAGITTAL WORKSPACE

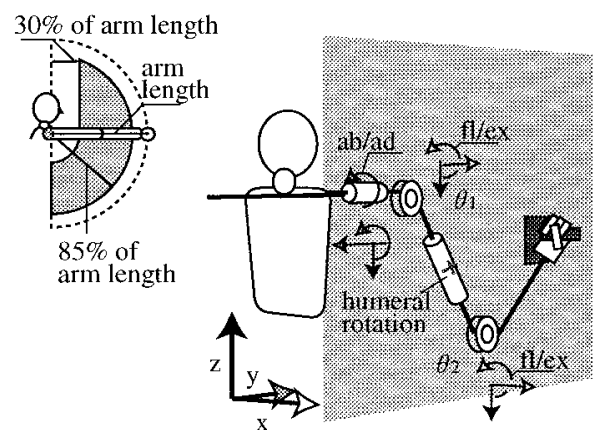

F VIA-POINT LOCATIONS
FIG. 3. Experimental setup, degrees of freedom for arm, and workspace. Subjects executed point-to-point movements on the horizontal board $(A)$ or sagittal board $(C)$. Movement within horizontal plane is performed by flexion/ extension (fl/ex) of elbow and horizontal $\mathrm{fl} / \mathrm{ex}$ of shoulder after abduction (abduction/adduction: ab/ad) with $90^{\circ}(B)$. Movement within sagittal plane is performed by fl/ex of elbow and shoulder $(D)$. Shaded area in top left figures included in $B$ and $D$ shows workspace where initial and final positions were located in horizontal and sagittal planes. Gray crosses on upper arm indicate humeral rotation is the same between horizontal and sagittal planes $(B$ and $D)$. $E$ : subjects performed via-point movements while watching a cathode ray tube (CRT) screen. $F$ : via-points indicated by asterisks and rounded by circles were used in early and late stages of training, and in training, respectively. in a chair, and their shoulders were fixed to the back of the chair with a harness. For reaching movements in the horizontal plane, the table was adjusted to lift the subjects' arms to shoulder level; the subjects' wrists were braced so that movement was constrained to the two degrees of freedom for the elbow and shoulder (Fig. 3A). Since the movements within the horizontal plane were performed at a slightly lower level than the shoulder level, a part of the hand and elbow was in contact with the surface. A semitransparent low-friction Teflon sheet covered the table. For movements in the sagittal plane, the subjects performed the experiment on a transparent acrylic board suspended along the sagittal plane passing through their right shoulders (Fig. $3 C$ ). The subject's elbow was constrained to be in the plane of hand movement because the abduction/ adduction of shoulder joint was restricted by the flat board. Hand movements were executed in this plane.

The subjects performed all of the trials with their right hands. The position of the hand was measured by the OPTOTRAK3020, which detected light-emitting diode signals from a marker. The marker was installed on a hand-held bar $(9 \mathrm{~cm}$ in height) perpendicular to the table. The sampling frequency was $200 \mathrm{~Hz}$. The subjects' task was to move their arms from an initial position to a final position (a circle with a radius of $2 \mathrm{~cm}$ ) shown on the plane within a time limit $(450-550 \mathrm{~ms})$; this was applied to the movement duration taken from the exit of the initial circle to the entrance of the final circle. The workspace was set within an annulus whose radius was between 30 and $85 \%$ of the arm length from the tip of the acromial process to the top of the hand-held bar (Fig. 3, B and D, top left shaded region).

The initial and final positions were randomly selected, and the distance of the movement was constant at $45 \%$ of arm length. The coordinates of each pair of initial and final points within the sagittal plane corresponded to those of the pair within the horizontal plane, with a $90^{\circ}$ shoulder abduction/adduction. First, the axis of abduction/ adduction is uniquely defined as the forward-backward direction vector (Cartesian $y$-axis in Fig. 3, $B$ and $D$ ), which is within the plane spanned by upper and lower arms. Second, the axis of flexion/ extension is defined uniquely so that it is orthogonal to the plane spanned by the upper and lower arms. Finally the axis of humeral rotation is the long axis of the upper arm (see Fig. 3, $B$ and $D$ ). By definition, the axis of humeral rotation and the flexion/extension axis are orthogonal with each other. Then, the pair of corresponding movements within the horizontal and sagittal planes use exactly the same shoulder and elbow flexion/extension freedoms. The articular limit used for these movements is different; however, many muscles that act on shoulder joint are shared in horizontal and sagittal movements. Hence the dynamic properties of these corresponding movements are very similar.

Fifty trials of 4 blocks for a total of 200 trials were performed within each plane. After each trial, the subjects were given feedback about whether their hands reached the target within a time limit. The subjects took a brief rest between blocks. 
In the second experiment, the subjects were one female $(C Y)$ and three males (HI, TY, and $A S), 27-33$ yr old. All subjects were right handed. In this experiment, we examined movements with longer duration and the larger variance of duration to determine the effect of duration on trajectory curvature. We lessened the limitation for duration adopted in the first experiment. We used a beep sound to indicate a standard beginning and end of movement, and the interval between sounds was $1 \mathrm{~s}$. The trials were all recorded only if the hand entered the target circle, so the variance of the movement duration was enlarged in comparison with the first experiment. The workspace was the same as the horizontal task in the first experiment, and the movement distance was $65 \%$ of the arm length. Each subject performed 50 trials.

VIA-POINT MOVEMENT. The subjects were two right-handed males ( $K H$ and $A H$ ) and one right-handed female $(N H), 20-23$ yr old. The setup of the measuring and experimental apparatus was the same as in the first horizontal experiment. A cathode ray tube (CRT) screen was placed in front of the subjects. Three circles and a cross were projected on this screen, which indicated the initial position, the viapoint, and the final position (with radii of 1,2 , and $2.5 \mathrm{~cm}$, respectively), and the current position of the hand measured by the OPTOTRAK (Fig. 3E).

If the via-point was set on the horizontal plane, the subjects would have frequently disturbed their visual field with their arms. To prevent occlusion of the via-point, we used a CRT screen. The subjects' task was to move their arms from the initial position to the final position by passing through a via-point within a time limit $(K H, 559-675 ; A M$, $570-690 ; N H, 525-635 \mathrm{~ms}$ ). The time limit of movement duration was decided according to the distance between the initial position (shoulder angle at $59^{\circ}$, elbow angle at $99^{\circ}$ ) and the final position (shoulder angle at $14^{\circ}$, elbow angle at $91^{\circ}$ ). The different initial and final positions and time limit are due to the different arm lengths of each subject. The via-point was extinguished at the onset of movement, which was indicated by a beep sound to avoid on-line correction of movements.

Eleven via-points were selected and equally arranged on the perpendicular bisector of the start-goal straight line (Fig. $3 F$ ). The combination of the initial position (open circle), final position (filled circle), and via point (asterisk) was regarded as a set. One of the 11 sets was randomly presented for each trial. First, the subjects performed 10 trials for each of the 11 via-points shown as asterisks in Fig. $3 F$ for a total of 110 trials (early stage of training). In the second task, the subjects were trained for five via-points shown as open circles in Fig. $3 F$ (training). Thirty trials were performed for each via-point, amounting to 150 trials. Finally, we carried out the third task to test the effects of training (late stage of training), where the number of trials and via-points were the same as in the early stage of training. The subjects obtained feedback concerning their individual hand paths and movement durations after each trial. The subjects took a brief rest between tasks.

\section{Filtering}

The position data were digitally filtered by a sixth-order Butterworth filter with an upper cutoff frequency of $10 \mathrm{~Hz}$. Derivatives of the position data were computed by applying a three-point local polynomial approximation. The actual beginning and end positions of each movement were determined using a two-dimensional curvature with a $3-\mathrm{mm}^{-1}$ threshold (Imamizu et al. 1995). Hence the movement duration, which was calculated from these start and end positions, was longer than the duration first required as a task condition.

For point-to-point movements, if the subject made a corrective movement, or the velocity at the end position was $>5 \%$ of the maximum velocity, the data were rejected as a failure. A trajectory with a velocity profile that deviated from an average two times larger than the standard deviation was taken out of the analysis as an outlier (see APPENDIX A for details).

\section{A WHOLE DEVIATION}

\section{$\mathrm{B}$}

COORDINATED ROTATION OF JOINTS
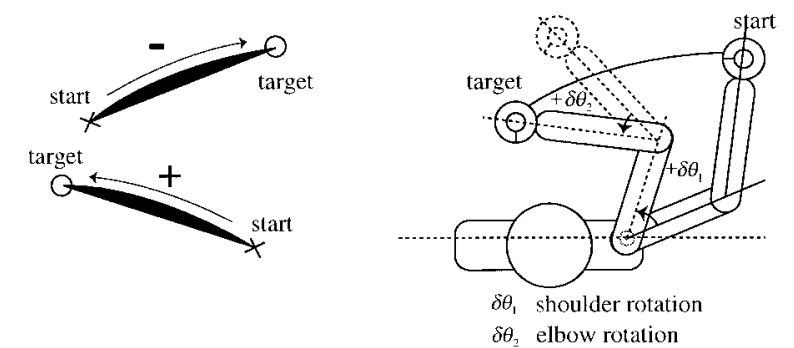

FIG. 4. Parameters that explain trajectory curvatures. A: filled black area is a "whole deviation" as index of trajectory curvature. The sign of a right curved trajectory for a vector from initial to final position is defined as positive, and the sign of a left curved trajectory is defined as negative. $B$ : coordinated rotation of joints is summation of quantity $\delta \theta_{1}$ that subtracts initial shoulder angle from final shoulder angle, and quantity $\delta \theta_{2}$ that subtracts initial elbow angle from final elbow angle.

\section{Analysis}

TRAJECTORY CURVATURES WITHIN THE HORIZONTAL AND SAGITTAL PLANES. Using data from the first experiment, we first investigated the correlation of trajectory curvatures between the horizontal and sagittal planes. The curvature of each trajectory was quantified as an area bounded by a start-to-goal straight line and the hand path (Fig. 4A). This area was named whole deviation; $W$ (Osu et al. 1997). The whole deviation concerned the direction in which the trajectory curved. If the trajectory curved right relative to the vector from start to target, the area was designated positive; on the other hand, a trajectory that curved left was given a negative sign. We examined the relationship between the curvatures of the paired trajectories within the horizontal and sagittal planes by calculating the correlation coefficients between horizontal and sagittal whole deviations. Here, the data utilized were from common trials in both planes adopted by the criteria mentioned above.

CONTRIBUTION OF THE COORDINATED ROTATION OF JOINTS. Next, the trajectory curvatures were linearly regressed from the sum of rotations of the elbow and shoulder joints from the initial position to the target (coordinated rotation of joints).

The coordinated rotation of joints $\delta_{\mathrm{c}}$ was defined by the following formulas

$$
\begin{gathered}
\delta_{\mathrm{c}}=\delta \theta_{1}+\delta \theta_{2} \\
\delta \theta_{1}=\theta_{1}\left(t_{\mathrm{f}}\right)-\theta_{1}\left(t_{0}\right) \\
\delta \theta_{2}=\theta_{2}\left(t_{\mathrm{f}}\right)-\theta_{2}\left(t_{0}\right)
\end{gathered}
$$

The rotation of shoulder joint $\delta \theta_{1}$ and that of elbow joint $\delta \theta_{2}$ denote the difference between final $\left[\theta_{1}\left(t_{\mathrm{f}}\right), \theta_{2}\left(t_{\mathrm{f}}\right)\right]$ and initial angles $\left[\theta_{1}\left(t_{0}\right)\right.$, $\left.\theta_{2}\left(t_{0}\right)\right]$ (Fig. $\left.4 B\right)$. $\delta_{\mathrm{c}}$ has a positive or negative sign when the joint is flexed or extended, respectively. Because the movement distances are constant, a large absolute $\delta_{\mathrm{c}}$ implies that two joints make large rotations in the same direction, e.g., transverse movement, whereas a small absolute $\delta_{\mathrm{c}}$ implies that two joints move in the opposite direction, e.g., radial movement. The whole deviations were linearly regressed from the coordinated rotations of joints by $\mathrm{Eq}$. 8 .

$$
W=a \delta_{\mathrm{c}}+b
$$

The coefficients $(a, b)$ and the square of correlation coefficients $\left(r^{2}\right)$ were calculated by the least-squares error method.

EXAMINATION OF MODELS. We compared measured trajectories with those predicted using each trajectory planning model. As constraints to simulate the trajectories for each model, we used the initial and final positions and movement durations determined from actual data. The velocity and acceleration were assumed to be zero at the 
initial and final positions. The minimum torque change trajectories and the minimum commanded torque change trajectories were computed by the steepest descent method (APPENDIX B). We compared measured and predicted trajectories for spatiotemporal properties. First, the correlations of whole deviations between both measured and predicted trajectories were compared. If the whole deviations of the measured trajectories completely corresponded to the predicted trajectories, the slope of the regression line fitted by the least-squares method would be 1.0, and the correlation coefficient ( $r$ ) would be 1.0. Second, the mean squared errors (MSE) were obtained for the position, velocity, acceleration, and torque between those measured and predicted as follows

$$
\frac{1}{N} \sum_{n=1}^{N}\left[\left(x_{n}^{\mathrm{raw}}-x_{n}^{\mathrm{model}}\right)^{2}+\left(y_{n}^{\mathrm{raw}}-y_{n}^{\mathrm{model}}\right)^{2}\right]
$$

where $n,\left(x_{n}^{\text {raw }}, y_{n}^{\text {raw }}\right),\left(x_{n}^{\text {model }}, y_{n}^{\text {model }}\right)$, and $N$ denote the number of 5-ms sampling points, the coordinates of actual data, those of predicted data, and the total sampling number, respectively. The MSEs allow trajectory comparisons including temporal variations. Bonferroni's $t$-test was used to test the differences among the models. If a certain model almost always gave the smallest MSEs, we considered that model to be statistically best for predicting actual trajectories.

CONTRIBUTION OF MOVEMENT DURATION. In the second experiment, whole deviations were linearly regressed from the coordinated rotation of joints and the signed movement duration $(T)$ as follows

$$
W=c \delta_{\mathrm{c}}+d T+e
$$

The movement duration was provided with the same sign as the whole deviation of each trajectory. Because similar results were obtained for all subjects to those in the first experiment, we combined the data of all of the subjects. If trajectory planning is based on the dynamic criteria, the movement duration was predicted to affect the hand trajectory (Uno and Kawato 1996).

CHANGE IN TRAJECTORY PROPERTY WITH TRAINING. For via-point movements, we focused on the transverse movement passing through a via-point in the front of the body. We analyzed trials where the hand entered the target circle within the time limit. For example, the hand did not always pass through a given via-point. Hence we divided the area among the nearest and furthest via-points into eight equal parts, and the hand trajectories that passed through each part of the area were averaged (Fig. 11, $A$ and $B$, area divided by dotted lines). The trajectory data were normalized by Atkeson and Hollerbach's (1985) method before averaging.

\section{RES ULTS}

\section{Trajectory curvatures within the horizontal and sagittal planes}

The averaged entire movement durations were $593 \pm 56$ (SD) $\mathrm{ms}, 561 \pm 51 \mathrm{~ms}$, and $550 \pm 57 \mathrm{~ms}$ in the horizontal plane and $620 \pm 55 \mathrm{~ms}, 644 \pm 53 \mathrm{~ms}$, and $634 \pm 60 \mathrm{~ms}$ in the sagittal plane using subjects in the order $M M, Y S$, and TT. The 191, 187, and 179 trajectories in the horizontal plane and 179, 176, and 179 trajectories in the sagittal plane, in that order, passed the criteria explained in METHODS (Filtering) and were used for further analysis.

In Fig. 5 we show samples of each of the five trajectories with large positive ( $B$ and $F$ ), small absolute $(C$ and $G$ ), and large negative ( $D$ and $H$ ) whole deviations. As previous research has demonstrated (Atkeson and Hollerbach 1985; Haggard and Richardson 1996; Uno et al. 1989a), hand trajectories are gently curved in some specific regions of the workspace, as
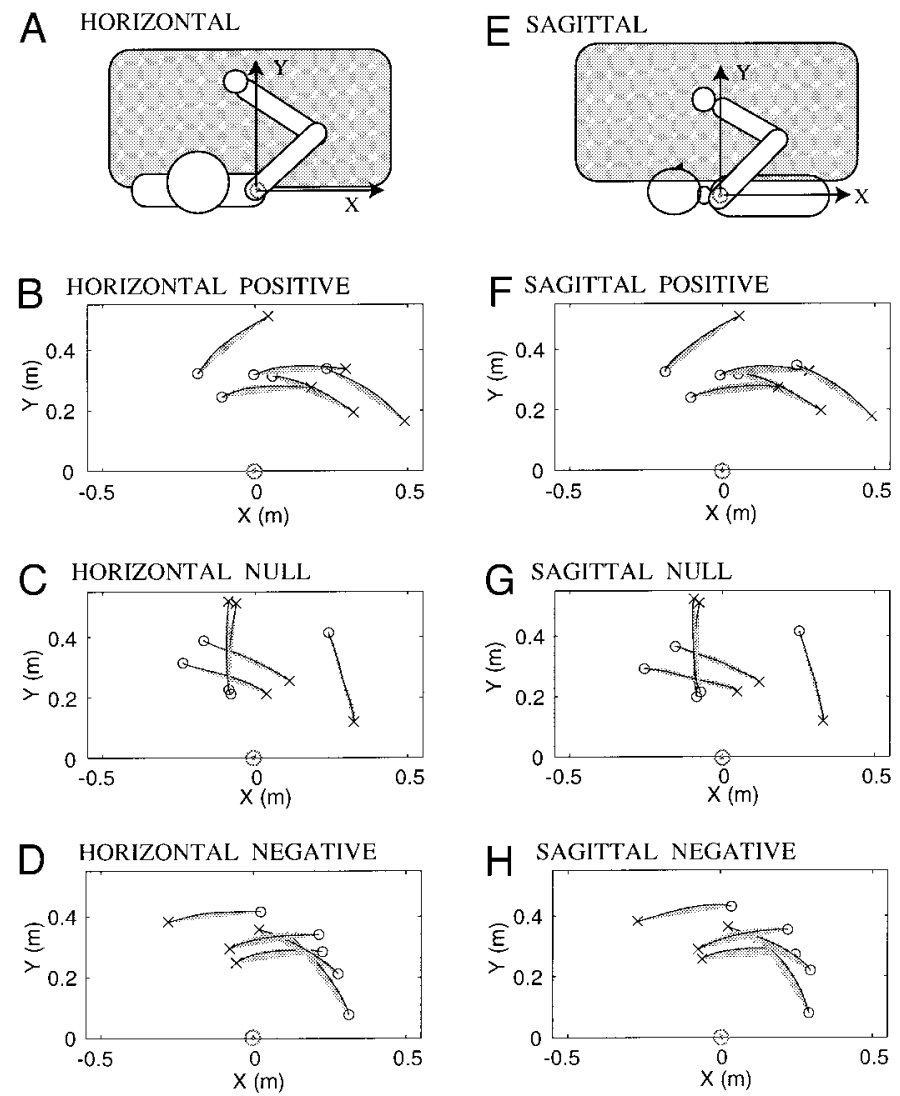

FIG. 5. $\quad A$ and $E$ indicate axes settings within horizontal and sagittal planes for $B-D$ and $F-H$ that show hand trajectories and reconstructed areas for model represented by the coordinated rotation of joints. $B$ and $F$ indicate 5 trajectories for positive whole deviations. $C$ and $G$ indicate 5 trajectories for small absolute whole deviations (straight line). $D$ and $H$ show 5 trajectories for negative whole deviations. Solid line is a measured hand trajectory, and shaded area below each trajectory is amount of reconstructed whole deviations by coordinated rotation of joints. For shaded area, actual path changes its shape by expanding or contracting related to perpendicular of start-goal straight line, so that its whole deviation becomes the same as that reconstructed by coordinated rotation of joints. Shaded area shows ability of coordinated rotation of joints to model the actual whole deviation. Linear model only predicts the whole deviation not any trajectory or path. $\times, \circ$, and $(0,0)$, the initial, final, and shoulder positions.

seen in this figure. On the horizontal plane, the hand paths of transverse movements appear to be convex toward the outside, whereas those of radial movements seem to be relatively straight. In the sagittal plane passing through the shoulder, paths for up-and-down movements are outwardly convex, and those for back-and-forth movements are relatively straight.

Significant positive correlations were obtained between the whole deviations of the hand trajectories in the horizontal and sagittal planes as shown in Fig. 6 (with subjects in the order $M M, Y S$, and TT: $r=0.75,0.65,0.86$; slope $=0.93,1.47,0.97$; $t(169,164,159)=14.59,21.50,11.11 ; P<0.001,0.001$, $0.001)$. The slopes of the regression lines fitted by the leastsquares method were almost 1 except for $Y S$. From these results, it was found that a pair of trajectory curvatures are similar when they correspond to each other within the horizontal and sagittal planes. However, a pair of trajectories that are different in the task space are identical with regards to the flexion/extension of the shoulder and elbow joints (see Fig. 3, $B$ and $D)$. Therefore the trajectory curvatures can be deter- 

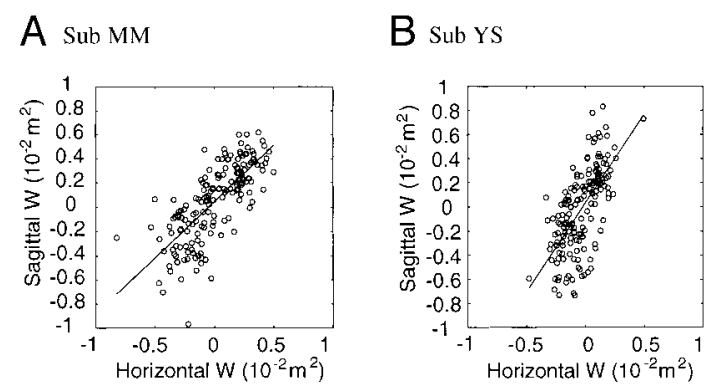

mined depending on the change of arm posture in the intrinsic body space. Furthermore, similar relationships were found between the horizontal and sagittal planes for whole deviations of the minimum commanded torque change trajectories (Fig. 7). Significant positive correlations with slope almost 1 were obtained between whole deviations predicted in the horizontal plane and those predicted in the sagittal plane for all of the subjects $(r=0.79,0.81,0.63$; slope $=1.12,1.02,0.87 ; t(169$, $164,159)=16.52,17.54,10.22 ; P<0.001,0.001,0.001)$. The slopes for actual data and predicted data indicated the same trend for the two subjects $M M$ and TT (Figs. 6 and 7) but not for subject $Y S$.

\section{Contribution of the coordinated rotation of joints}

Table 2 summarizes the results of the linear regression of the whole deviation from the coordinated rotation of joints for all subjects. The regressions were significant, and squared correlation coefficients were high for all subjects (the mean $r^{2}$ in the order of the horizontal and sagittal planes: $0.75 \pm 0.14,0.73 \pm$ 0.06). In Fig. 5, the shaded area below each trajectory represents the amount of reconstructed whole deviations by the coordinated rotation of joints (data from $T T$ with the highest $r^{2}$ ). Note that this linear model only predicts the whole deviation and not any trajectory or path. The reconstructed whole deviations closely simulate the actual whole deviations concerning magnitudes and directions. The positive coefficient values in $a$ of $E q .8$ statistically confirm that trajectories having large positive whole deviations appeared in right-to-left and down-to-up movements (Fig. 5, $B$ and $F$ ), and those having large negative whole deviations were mainly observed in leftto-right and up-to-down movements (Fig. 5, $D$ and $H$ ). This also suggests that trajectories having small whole deviations were found in radial and fore-and-aft movements in the polar coordinates of the shoulder (Fig. 5, $C$ and $G$ ). These results indicated that trajectory curvatures can be reproduced well from the coordinated rotation of joints related to dynamics, as discussed later.
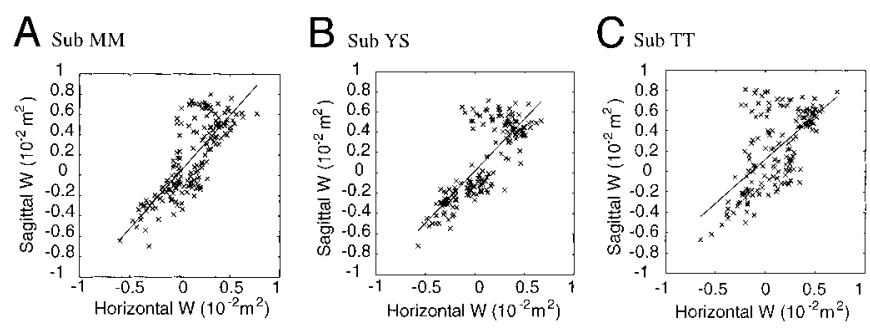

FIG. 7. Correlations of whole deviations predicted by minimum commanded torque change model in horizontal and sagittal planes of all subjects. A: subject MM. B: subject YS. C: subject TT.

\section{Examination of models}

Figure $8 A$ shows the trajectories measured and predicted by each criterion in the horizontal plane. In comparison with actual trajectories, many of the minimum angle jerk trajectories and minimum torque change trajectories were largely curved toward the outside and inside of the body, respectively. The same tendencies were shown for data measured in the sagittal plane. The velocity ( $B$ and $C$ ), acceleration $(D$ and $E$ ), and torque profiles $(F$ and $G)$ of the sample movement denoted by the arrows are shown in Fig. 8. The predictions of the minimum commanded torque change model were best fitted to data relating to all properties, namely, velocity, acceleration, and torque.

The correlations between the whole deviations of measured and predicted trajectories, and regression lines are indicated in Fig. 9. Table 3 summarizes the correlation coefficients, the results of a test on the correlations, and the slopes of the regression lines of all subjects in the horizontal and sagittal planes. The correlation coefficients for the three models were significant, except for the minimum hand jerk model. All of the minimum hand jerk trajectories were straight, so they were not correlated with those of actual trajectories (the mean correlation coefficient: 0 , mean slope: 0 ). It was quantitatively indicated that the minimum angle jerk trajectories were curved larger than actual trajectories (in horizontal and sagittal planes, the mean correlation coefficients, 0.75 and 0.76 ; mean slopes, 2.0 and 1.35). Most of the whole deviations of the minimum torque change trajectories had negative correlations to those of actual trajectories, and the correlation coefficients were low (the mean correlation coefficients, -0.38 and -0.36 ; mean slopes, -1.09 and -0.39 ). The whole deviations of the minimum commanded torque change trajectories were smaller than, or approximately the same as, those of actual trajectories, and the correlation coefficients were high (the mean correlation

TABLE 2. Contribution of the coordinated rotation of joints

\begin{tabular}{lccc}
\hline \hline Subjects & $r^{2}$ & $a$ & $b$ \\
\hline & \multicolumn{3}{c}{ Horizontal } \\
$M M$ & 0.75 & $0.32^{*}(566.59)$ & -2.66 \\
$Y S$ & 0.54 & $0.16^{*}(219.62)$ & -3.15 \\
$T T$ & 0.81 & $0.49^{*}(732.20)$ & -3.06 \\
& & & \\
& & Sagittal & 1.47 \\
$M M$ & 0.69 & $0.39^{*}(390.98)$ & -2.18 \\
$Y S$ & 0.69 & $0.41^{*}(387.08)$ & -3.45 \\
$T T$ & 0.80 & $0.55^{*}(713.74)$ & \\
\hline
\end{tabular}

Curvatures were regressed linearly from the coordinated rotation of joints. $F$ values are in parentheses. $* P<0.01$. 
A

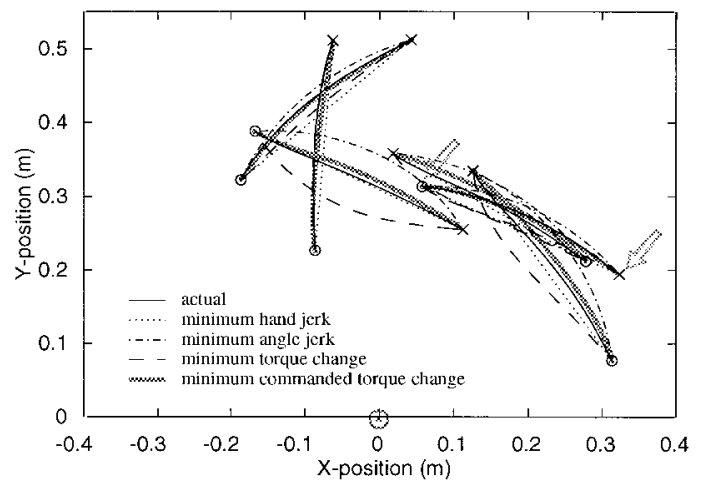

B

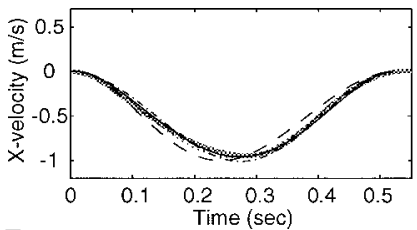

D

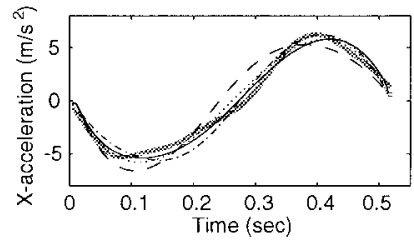

F

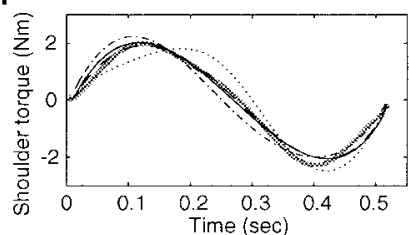

C

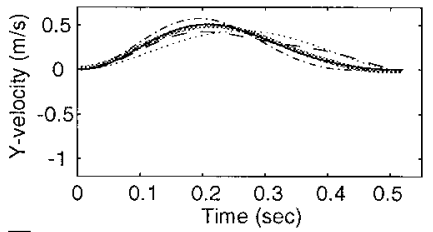

$\mathrm{E}$

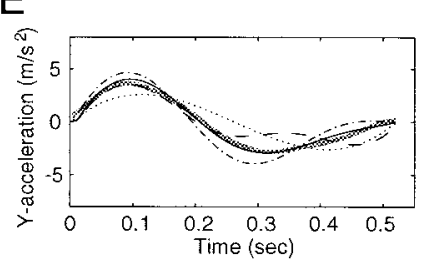

G

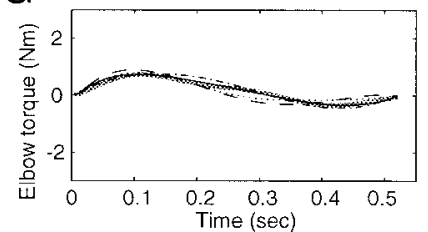

FIG. 8. Examples of trajectory properties measured and predicted by minimum hand jerk, minimum angle jerk, minimum torque change, and minimum commanded torque change models are shown by solid gray lines, dotted lines, dash dot lines, broken lines, and solid lines, respectively. $A$ : paths. $B$ and $C$ : velocities. $D$ and $E$ : accelerations. $F$ and $G$ : torques. $\times, \circ$, and $(0,0)$, the initial, final, and shoulder positions. Profiles shown in $B-G$ are derived from trajectories whose initial and final positions are indicated by arrows in $A$.

coefficients, 0.70 and 0.80 ; mean slopes, 0.80 and 0.84 ). In subject TT, the minimum angle jerk model has a better correlation than the minimum commanded torque change model in the horizontal plane, however, the scattered whole deviations to right and left as shown in Fig. 9, $C$ and $D$, imply that the minimum angle jerk model cannot predict small whole deviations observed in actual trajectories. This tendency is common in all subjects.

We carried out $t$-tests for the MSEs of the position, velocity, acceleration, and torque of the trajectories measured in both planes. The histograms of the MSEs of torques are shown in Fig. 10. In 72 total comparisons between the minimum commanded torque change model and other three models, four characteristics, three subjects, and two planes $(3 \times 4 \times 3 \times 2)$, the MSEs of the minimum commanded torque change model for 71 (66) comparisons were smaller (significantly smaller, $P<0.05)$. We roughly summarized for the MSEs that the minimum commanded torque change model was the best, the

minimum hand jerk model the second best, the minimum angle jerk model the third best, and the minimum torque change model the worst. In the experiments in the horizontal and sagittal planes, we found that the minimum commanded torque change model quantitatively and statistically predicted trajectories the best.

\section{Contribution of movement duration}

A total of 190 trajectories was used in the analysis. The average duration of movement was $688 \pm 131 \mathrm{~ms}$. Table 4 summarizes the results of linear regression calculated using $E q$. 10. The regression was significant and the squared correlation coefficient was high. These results quantitatively indicated that the trajectory curvatures were dependent on the duration of movement and the locations of the initial and final points quantified by the coordinated rotation of joints. Moreover, these results support the prediction of Uno and Kawato (1996)
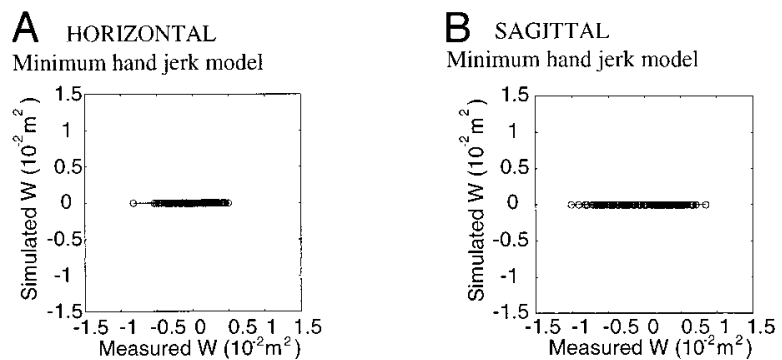

\section{HORIZONTAL}

Minimum angle jerk model

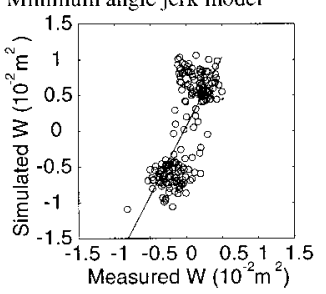

D SAGITTAL

Minimum angle jerk model

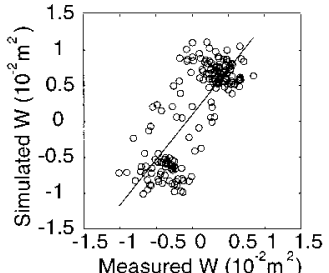

E HORIZONTAL

Minimum torque change model

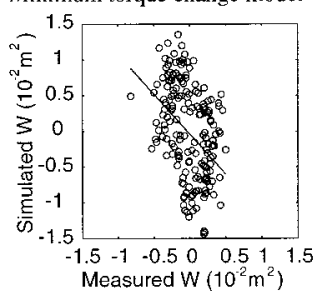

F sagitTal

Minimum torque change model

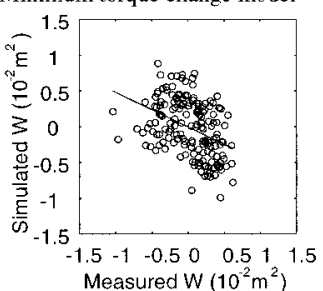

G hORIZONTAL

H SAGITTAL

Minimum commanded torque change model
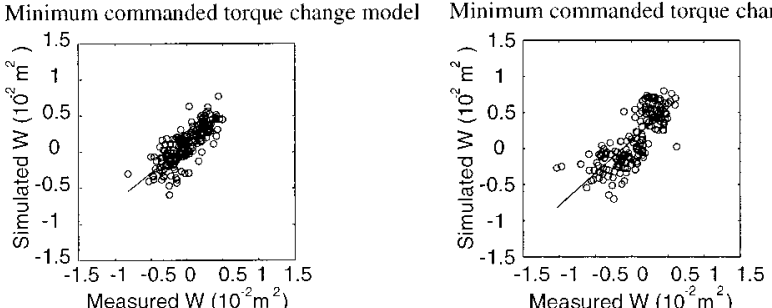

FIG. 9. Correlations of measured whole deviations and predicted whole deviations within horizontal plane $(A, C, E$, and $G)$ and sagittal plane $(B, D, F$, and $H$ ) are shown for each model. $A$ and $B$ : minimum hand jerk model. $C$ and $D$ : minimum angle jerk model. $E$ and $F$ : minimum torque change model. $G$ and $H$ : minimum commanded torque change model. Data from subject $M M$. 
TABLE 3. Relationship between measured and predicted whole deviations

\begin{tabular}{|c|c|c|c|c|c|c|c|c|c|c|c|c|}
\hline \multirow[b]{3}{*}{ Models } & \multicolumn{12}{|c|}{ Subjects } \\
\hline & \multicolumn{4}{|c|}{$M M$} & \multicolumn{4}{|c|}{$Y S$} & \multicolumn{4}{|c|}{$T T$} \\
\hline & $r$ & $t$ & $d f$ & Slope & $r$ & $t$ & $d f$ & Slope & $r$ & $t$ & $d f$ & Slope \\
\hline \multicolumn{13}{|c|}{ Horizontal } \\
\hline HJ & 0 & & & 0 & 0 & & & 0 & 0 & & & 0 \\
\hline $\mathrm{AJ}$ & 0.73 & $14.56^{*}$ & 189 & 1.93 & 0.68 & $12.62^{*}$ & 185 & 2.65 & 0.84 & $20.17^{*}$ & 177 & 1.44 \\
\hline $\mathrm{TC}$ & -0.39 & $5.78^{*}$ & 189 & -1.12 & -0.35 & $5.02 *$ & 185 & -1.45 & -0.40 & $5.81^{*}$ & 177 & -0.71 \\
\hline CTC & 0.79 & $17.45^{*}$ & 189 & 0.80 & 0.68 & $12.69 *$ & 185 & 1.28 & 0.64 & $10.95^{*}$ & 177 & 0.47 \\
\hline \multicolumn{13}{|c|}{ Sagittal } \\
\hline HJ & 0 & & & 0 & 0 & & & 0 & 0 & & & 0 \\
\hline AJ & 0.75 & $15.12 *$ & 177 & 1.49 & 0.73 & $14.20 *$ & 174 & 1.28 & 0.79 & $17.04 *$ & 177 & 1.27 \\
\hline TC & -0.40 & $5.81 *$ & 177 & -0.49 & -0.32 & $4.49^{*}$ & 174 & -0.30 & -0.39 & $5.11^{*}$ & 177 & -0.38 \\
\hline CTC & 0.78 & $16.65^{*}$ & 177 & 0.90 & 0.83 & $19.78^{*}$ & 174 & 0.91 & 0.77 & $16.65^{*}$ & 177 & 0.71 \\
\hline
\end{tabular}

$r$, correlation coefficient; $t, t$ values; $d f$, degrees of freedom; HJ, minimum hand jerk model; AJ, minimum angle jerk model; TC, minimum torque change model; CTC, minimum commanded torque change model. $* P<0.01$.

that longer movement duration causes larger trajectory curvature. We will explain this prediction later in the paper.

\section{Change in trajectory property with training}

The number of trajectories adopted for analysis were 52, 61, and 48 for the early stage of training, and 68, 73, and 62 for the late stage of training for subjects in the order $K H, A M$, and $N H$. The average duration of movement was $951 \pm 96 \mathrm{~ms}, 901 \pm$ $140 \mathrm{~ms}$, and $847 \pm 59 \mathrm{~ms}$ for the early stage of training, and $878 \pm 80 \mathrm{~ms}, 883 \pm 89 \mathrm{~ms}$, and $863 \pm 58 \mathrm{~ms}$ for the late stage of training in the same order.

Figure 11 shows the mean paths and tangential velocity profiles measured $(A, B, D$, and $E$; data from subjects $A M$ and $N H$ ) and those predicted for each model of subject $A M$ ( $C$ and $F)$. The trajectories were predicted using the experimentally specified initial position, final position, via-point, and averaged movement duration of the measured data from subject AM. To clearly show the variety of tangential velocity profiles depending on the via-points locations, we changed the amplitudes of
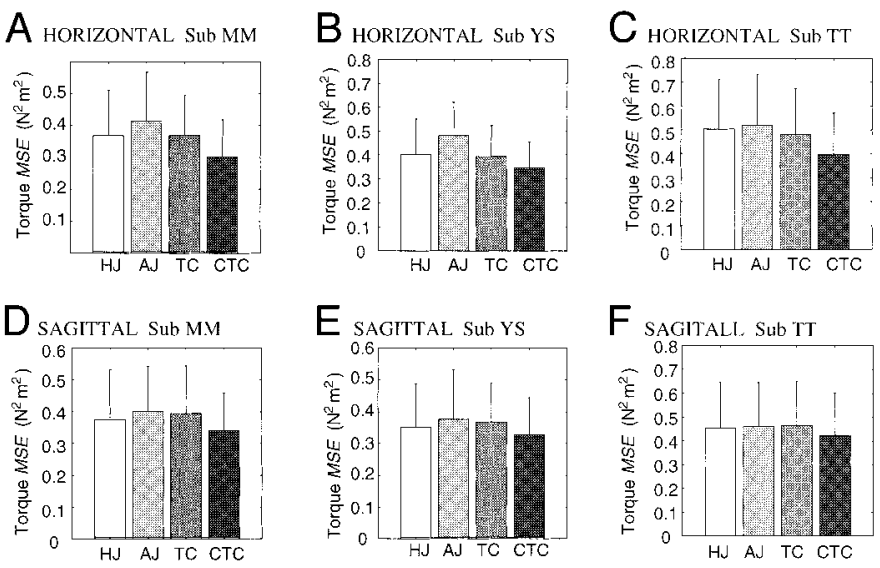

FIG. 10. Mean \pm SD of squared errors of torques measured and predicted within horizontal plane $(A-C)$ and sagittal plane $(D-F)$ for each subject. $A$ and $D$ : subject MM. B and E: subject YS. $C$ and $F$ : subject TT. HJ, AJ, TC, and $\mathrm{CTC}$, minimum hand jerk model, minimum angle jerk model, minimum torque change model, and minimum commanded torque change model, respectively. Error bars indicate SD. velocity profiles corresponding to the deviations between the start-goal straight line and via-points in Fig. $11(D-F)$. When a via-point was set near the body with respect to start-goal line, the deviation was designated negative. For example, a bottom path $(A, l e f t)$ and a bottom profile $(D$, left $)$ were derived from common data.

The mean paths changed to asymmetric shapes on the right and left in the late stage of training in comparison with the relatively symmetric shapes in the early stage of training (Fig. $11 A)$. Note that the minimum hand jerk model predicts symmetric shapes of the path, whereas all other models predict asymmetric shapes.

In the data from subject $A M$, the tangential velocity profiles had double peaks in the early stage of training, both in trials with a via-point far from and near to the body. However, in the late stage of training, a double peak appeared only when a via-point was near to the body, and the time at maximum velocity changed from earlier to later as the via-point shifted toward the body. According to the data from subject $\mathrm{NH}$, the double peaks of the tangential velocity profiles, which were not clearly observed in the early stage of training, were solely visible in near via-points in the late stage of training. For almost all data, the single peaks observed for far via-points after learning are also observed at the single trial level (33/36, $38 / 38$, and $33 / 37$; number of single-peak trials/number of adopted trials in the order $A M, N H$, and $K H$ ). The time at maximum velocity in the late stage of training was different from the early stage of training. This time changed from the middle of the movement duration to earlier in the far viapoints. For all subjects, tangential velocity profiles appeared to

TABLE 4. Contribution of movement duration

\begin{tabular}{cccc}
\hline \hline \multicolumn{4}{c}{ Coefficients } \\
\hline$r^{2}$ & $c$ & $d$ & $e$ \\
\hline 0.71 & $0.22 *(11.72)$ & $0.07 *(53.30)$ & 6.09 \\
\hline
\end{tabular}

Signed curvatures were regressed linearly from the coordinated rotation of joints and the signed movement durations. $F$ values are in parentheses. ${ }^{*} P<$ 0.01 . 
A AVERAGED PATHS: Sub AM
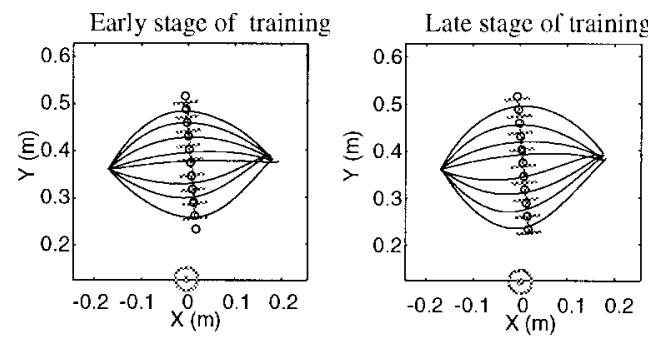

\section{PREDICTED PATHS: Sub AM}
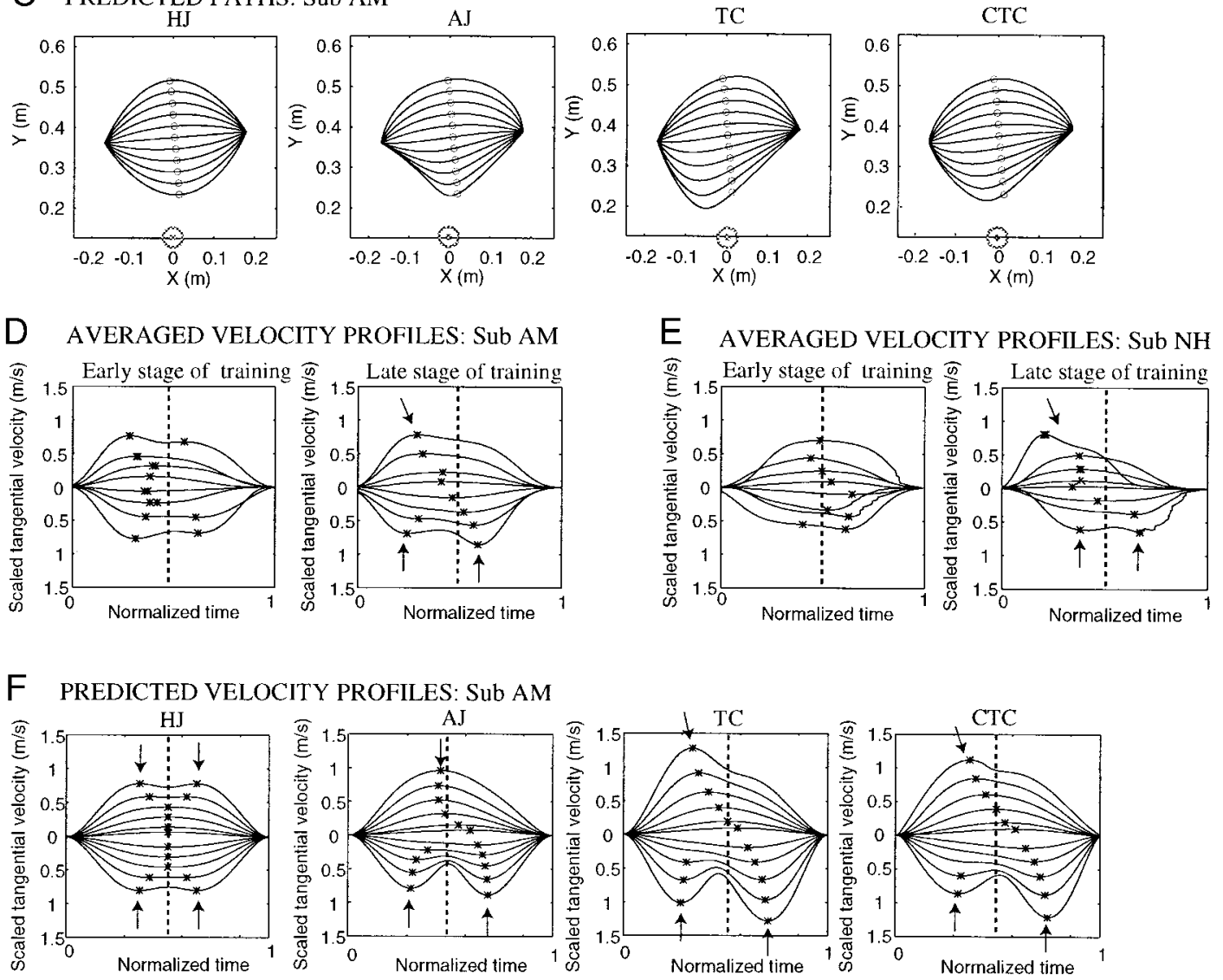

FIG. 11. Averaged hand paths $(A$ and $B)$ and tangential velocity profiles $(D$ and $E$ ) for each subject. $A$ and $D$ : subject $A M . B$ and $E$ : subject $N H$. Hand paths $(C)$ and tangential velocity profiles $(F)$ predicted by each model. $\bigcirc$, an experimentally designated via-point in $A-C$. *, Peak of velocity in $D-F$. Magnitudes for each velocity profile were weighted by the following values; $D, 1.0$, $0.8,0.5,0.2,0.1,0.4,0.7$, and 1.1 in early stage of learning, $1.0,0.8,0.5,0.2,0.4,0.7$, and 1.1 in late stage of learning; $E, 1.0$, $0.7,0.4,0.1,0.2,0.5,0.8$, and 1.1 in early stage of learning, $1.0,0.7,0.5,0.2,0.1,0.3,0.6$, and 0.9 in late stage of learning; $F$, $1.0,0.8,0.6,0.4,0.2,0.1,0.2,0.4,0.6,0.8$, and 1.0 from top to bottom, respectively. To obtain values used in $D$ and $E$, each distance from intersection of averaged path and the perpendicular bisector of start-end straight line to start-end straight line was divided by distance between furthest intersection from body and start-end straight line. Values used in $F$ were obtained by dividing each distance from via-point to start-end straight line by distance from a top via-point to the start-end straight line. Values of the 5th profile from the top in early stage of training of subject $A M(D)$ and middle profiles in $F$, however, were changed because zero values were obtained by above method.

be more asymmetric in shape on the right and left in the late stage of training than in the early stage of training when via-points were set further from the body. In the late stage of training, the characteristics of the actual tangential velocity profiles for all subjects stated above were very similar to those of the minimum torque change trajectories and the minimum commanded torque change trajectories (Fig. 11, $D-F$ ).

We do not show the data of subject $K H$, because we could not observe any training effects. However, the minimum torque change and minimum commanded torque change models re- produced the paths and tangential velocity profiles of actual data well. It should be noted that subject $K H$ had experience with via-point movements in a different experimental design, so he already possessed some skills.

\section{I S C U S S I O N}

We first discuss the relationship between the minimum commanded torque change model and the minimum jerk model of equilibrium trajectory (Flash 1987) rather than the actual tra- 
jectory. The two models are close in the sense that smoothness is imposed on representations related to muscle elastic characteristics. The space of trajectory planning for the minimum jerk model of equilibrium trajectory is considered to be more centrally located in the brain from the visual input side than that of the original minimum hand jerk model because the former is related to a form of motor command (equilibrium trajectory), whereas the latter is defined in a purely sensory visual space. Similarly, the minimum commanded torque change model is more central from the motor output side than is the literal minimum torque change model. Thus the two approaches starting from the input and output extremes seem converge to intrinsic-dynamic-neural representations for trajectory planning. However, conceptual differences still exist between the two approaches. The planned trajectory itself takes into account the dynamics of the muscle, arm, and the environment in the minimum commanded torque change model, but not in the minimum jerk model of equilibrium trajectory. A recent model by Harris and Wolpert (1998) can be classified as a version of the minimum motor command change model.

In the present experiments, we adopted initial position, final position, and movement duration as the independent variables to examine trajectory curvature. Movement distance is an additional plausible independent variable. Because an enormous number of trajectories are needed to be measured if all of four parameters were to be set as independent variables, the number of parameters should be limited. The most influential parameters in trajectory curvatures were found the initial and final positions. As shown in the experiment by Uno et al. (1989a) and our experiments, the movement duration only modestly affects the trajectory curvature. Furthermore, in preliminary experiments, we found that variations in movement amplitude lead to little change in curvature. Because we examined the three most influential variables in trajectory curvatures out of four variables, we may claim that our experiments add considerable generality to the previous work.

\section{Trajectory curvatures within the horizontal and sagittal planes}

We first explored whether the trajectory is planned in extrinsic or intrinsic coordinates by comparing trajectory curvatures between the horizontal and sagittal planes. Movements in the horizontal and sagittal planes can be executed by the same extension/flexion of elbow and shoulder joints. In this respect, each pair of movements in both planes dynamically corresponds to each other. The coordinated rotations of joints among the corresponding movements were the same. For example, the left-to-right transverse movement in the horizontal plane was equivalent to the downward up-and-down movement in the sagittal plane.

We examined the correlation between whole deviations of hand trajectories in the horizontal plane and in the sagittal plane. There was a variety of trajectory curvatures measured at various locations, and the trajectories of corresponding pairs tended to be curved with the same direction and amplitude. This suggests a strong relationship between the trajectory curvatures and variables in the body space such as flexion/extension.

Furthermore, the correspondence of curvatures in both planes suggest a weak gravitational influence on movements, and thus common properties of hand trajectories achieved with the same coordinated rotations of joints. Such common features were found in the predicted trajectories by the minimum commanded torque change model. Moreover, similar slopes of actual data (Fig. 6) and model predictions (Fig. 7) obtained from two of three subjects suggest that this model can predict a trajectory that has almost the same curvature as the actual trajectory. The movements within both planes have the same kinematic variables (coordinated rotation of joint) in the intrinsic coordinates; however, kinematic variables in the extrinsic coordinates are not the same between these planes. Hence it seems that this equivalence supports planning in intrinsic space but not extrinsic space. However, we cannot discuss if the trajectory is planned in dynamic space or kinematic space from these results.

The dynamic model can predict different trajectories for the different planes because it depends on gravity. On the other hand, the kinematic model is not influenced by gravity. In the present experiments, we assumed that gravity had little influence on movements within the horizontal plane, because the subjects' arms were supported by a table. For movements within the sagittal plane, gravity should be considered because of the lack of support. Even in this case, the hand trajectory was found to be quite insensitive to weights held during movements both in the real data and the theoretical calculation (Atkeson and Hollerbach 1985; Uno et al. 1989a). That is, a small effect of weights was observed on the optimal trajectories predicted by the original minimum torque change model (Uno et al. 1989a). Even if that trajectory is generated based on dynamic criteria, the hand trajectory is apparently not much affected by gravity because its influence is almost spatially uniform and thus not large enough to change the optimal trajectory. This is the theoretical background why the dynamic model such as the minimum commanded torque change model can reproduce similar curvatures shown in the trajectories measured within the horizontal and sagittal planes.

Wolpert et al. (1994) proposed the effect of visual perceptual distortion on trajectory planning to explain the gently curved hand trajectories observed in transverse movements. Conversely, Osu et al. (1997) demonstrated that hand trajectories are also curved within the frontoparallel plane for which the visual perceptual distortion is negligible (Foley 1980; Indow and Watanabe 1988). We assume that the visually distorted lines within the horizontal and sagittal planes are different from each other. If the idea of Wolpert et al. (1994) was appropriate, we would find different curvatures between the actual trajectories measured in these planes. The experimental results here showed almost the same trajectory curvatures measured in these planes as mentioned above for two of three subjects. Our outcome may be considered as further counterevidence against the effect of visual perceptual distortion on trajectory planning.

\section{Contribution of the coordinated rotation of joints}

We were able to quantitatively demonstrate that trajectory curvatures are dependent on the coordinated rotation of joints. In a large coordinated rotation of joints, namely a transverse movement when the shoulder and elbow rotate toward the same direction, the interactional torques such as off-diagonal components of inertial matrices and centrifugal forces increase with the same signs; therefore the torque around the joint is 
enlarged. In contrast, when the coordinated rotation of joints is small, the force components related to the shoulder and elbow joints have the inverse signs of each other, and torque is resultingly small. Therefore the coordinated rotation of joints is closely connected to the torque generated around the joint. We can say that the coordinated rotation of joints is a kinematic variable, but it is closely related to the arm dynamics.

\section{Examination of models}

For movements within the horizontal and sagittal planes, the minimum commanded torque change model was able to reproduce the spatial characteristics of measured trajectories, in this case, the magnitudes and directions of curvatures, better than the other three models. The minimum torque change model could neither reproduce the magnitudes nor the directions of curvatures. The minimum hand jerk model, which always predicts straight paths, showed a lack of correlation with the measured trajectories regarding the whole deviations. Even though the minimum angle jerk model could explain the direction of the curvature, it predicted trajectories with obviously excessive curvatures.

In previous studies, Hollerbach (1990) and Osu et al. (1997) suggested that planning in the joint space cannot explain a gently curved hand trajectory. As shown in Fig. 9, the slopes relating whole deviations of the minimum hand jerk and actual trajectories were zero (the mean error of the slope was 1 in both planes). For minimum angle jerk trajectories, slopes were from 1.4 to 2.7 in the horizontal plane and from 1.3 to 1.5 in the sagittal plane (the mean error of the slope was 1 and 0.35 , respectively). Hence it can be considered that the minimum angle jerk model is quantitatively three times better than the minimum hand jerk model in the sagittal plane. Although the minimum angle jerk model has a qualitative weak point in predicting trajectories that are too curved, it is quantitatively a better model compared with the minimum hand jerk model. Accordingly, we demonstrated that it is impossible to completely reproduce actual data with trajectory planning in the kinematic space. As Flash (1990) pointed out, we confirmed that literal minimum torque change trajectories, computed without consideration of the viscosity, cannot reproduce any actual trajectory.

\section{Contribution of movement duration}

Uno and Kawato (1996) predicted that longer movement duration causes a viscous term to be relatively larger than the other terms, namely, inertia, centrifugal force, and the Coriolis force. Uno and Kawato (1996) reported that a trajectory is curved toward the outside (right side) with high viscous values, and low viscous values lead the trajectory to curve inside (left side). Mathematically, the original minimum commanded torque change trajectory, which is computed by multiplying the movement duration by $\lambda$, is the same as that computed by multiplying viscosity coefficients by $\lambda$ without changing the movement duration (Uno and Kawato 1996). Therefore a longer movement duration can be predicted to cause a more curved trajectory. This was confirmed in behavioral experiments (Uno and Kawato 1996). The results of our second experiment quantitatively supported these previous studies with an enormous amount of data.
Movements performed in various places within a plane may have different levels of difficulty. If a difficult movement leads to a highly curved trajectory, we consider the possibility of the movement duration being long due to this difficulty. In this case, we cannot discuss the contribution of the movement duration to the trajectory curvature because the movement duration and the coordinated rotation of joints are correlated variables. Therefore we performed the following analysis solely to investigate the influence of the movement duration.

$$
\begin{gathered}
W_{\text {predicted }}=f \delta_{\mathrm{c}}+g \\
\delta W=W-W_{\text {predicted }}
\end{gathered}
$$

The whole deviations $W$ are first linearly regressed using only the coordinated rotation of joints. That is, $W_{\text {predicted }}$ are reconstructed by the coefficient $f$ and the intercept $g$, which were obtained by $E q$. 11. Then we obtain $\delta W$ by subtracting $W_{\text {predicted }}$ from $W$. This value is regarded as the amount that cannot be explained by coordinated rotation of joints. We examined the correlation of $\delta W$ and the movement duration. The result showed significant positive correlations $(r=0.35$, $t=5.10, P<0.001)$. Hence it was statistically confirmed that curvature of hand trajectory is dependent on the movement duration.

\section{Change in trajectory property with training}

For via-point movements, we averaged paths and tangential velocities using measured data and compared them with the paths and tangential velocities predicted by each model. The measured trajectory properties were closer to those predicted by the minimum torque change model, the minimum commanded torque change model, and the minimum angle jerk model with training. However, the minimum torque change model and minimum angle jerk model could not reproduce the characteristics of actual trajectories in the first experiment. Because the minimum commanded torque change model was able to accurately explain data measured for both point-topoint movements and via-point movements, this model seemed to be the best of the four models. The results of the present experiments suggested that this model is applicable to skilled rather than to novel movements.

Minimizing the commanded torque change or the motor command change is the implicit constraint, and the constraints for the target, movement duration, and via-point are explicit. The latter constraints are given from the external world, whereas the former constraints are not. Even if the hand path, velocity, and acceleration of movements are provided as the explicit constraints, the CNS will have to finally compute torques or motor commands capable of meeting these constraints. The subjects can meet the implicit constraints such as minimizing the commanded torque change while meeting these explicit constraints in the process of training. There is also the possibility of adopting a different criterion to execute skilled or unskilled movements. In either case, the CNS seems to have the tendency to finally learn to perform optimization in the higher space, e.g., the commanded torque or motor command space.

The actual properties of paths and tangential velocities in the late stage of training converged with those predicted by the minimum commanded torque change model. This model could 
explain the properties of hand trajectories for both point-topoint movements and via-point movements. Taking all of these results together obtained in the present experiments, we were able to suggest that hand trajectory is planned in the intrinsic coordinates considering arm and muscle dynamics and using representations for motor commands controlling muscle tensions.

\section{APPENDIX A}

\section{Dissimilarity index of velocity profiles}

We quantified the tangential velocities of all of the data by normalizing the trajectories for the duration and distance of the movement (Atkeson and Hollerbach 1985). The mean of these normalized tangential velocity profiles was regarded as the reference profile. We calculated the maximum common area among areas surrounded by a given normalized tangential velocity profile and the $x$-axis, and areas bounded by the reference profile and the $x$-axis. The whole area surrounded by these two profiles was divided into the common part (Fig. A1, light shaded area) and noncommon part, that is, the summation of the differences between a normalized profile and the reference profile at each time instance (dark shaded area). Then the ratio of the noncommon area to the whole area was determined for each trajectory. We defined this ratio as a dissimilarity index of the reference profile and actual profile as shown below.

\section{APPENDIX B}

\section{Calculation of optimal trajectories}

The minimum torque change and minimum commanded torque change trajectories were computed by combining the steepest descent method and penalty method. The objective function is defined as

$$
L=\left(\theta_{\mathrm{f}}-\theta_{\mathrm{f}}^{\mathrm{d}}\right)^{2}+\left(\dot{\theta}_{\mathrm{f}}-0\right)^{2}+\lambda / 2 \int_{t_{0}}^{t_{\mathrm{f}}}(\mathrm{d} \tau / \mathrm{d} t)^{2} \mathrm{~d} t
$$

$\tau$ was varied to $-\partial L / \partial \tau$ to minimize $L$ (gradient method). Here, the first term of $L$ denotes the squared error of the joint angle at the final position of predicted movement $\theta_{\mathrm{f}}$ and that of desired movement $\theta_{\mathrm{f}}^{\mathrm{d}}$, and requires the arm to reach a target. The second term demands that the velocity at the final position is zero. The third term shows that the objective function of the minimum torque change is multiplied by $\lambda$, such that it requires minimization of the torque change. By gradually decreasing $\lambda$ from a large value (penalty method), the trajectory and torque, of which the torque change is the smallest, can be calculated with the condition that the hand reaches the target. We can obtain some resolution with this method because of its stability; however, there is no guarantee that the resolution is optimal. If the reduction of

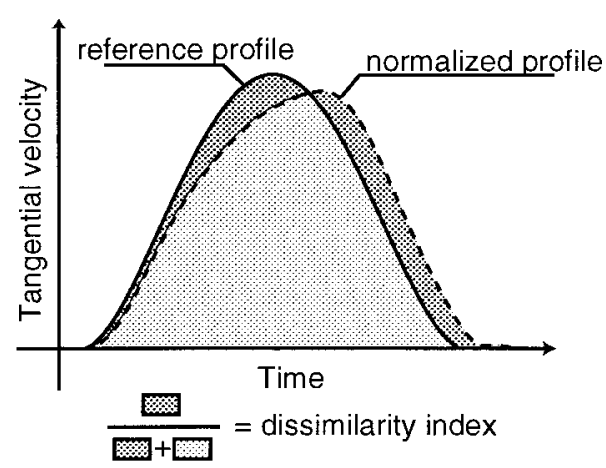

FIG. A1. Quantification of deviation of each trajectory from the normalized trajectory. $\lambda$ is too early or the change of $\tau$ is insufficient, a trajectory may be obtained in which the torque change is not smooth.

For instance, we set the initial $\lambda$ to 500 and performed 10,000 repetitive computations for each of 9 sets, namely, a total of 90,000 times to compute the minimum commanded torque change trajectories in the horizontal task of the first experiment. The value of $i+1$-th $\lambda$ at the $N$ th set was calculated by the following equation

$$
\begin{gathered}
\lambda_{i+1}^{N}=\lambda_{i}^{N}-\left(g \lambda \cdot \lambda_{1}^{N} / i t r\right) \\
g \lambda=0.9
\end{gathered}
$$

where $i t r$ expresses the repetition number of times for each set. $\lambda_{1}^{N}$ denotes the initial value of $\lambda$ at the $N$ th set.

This study was partially supported by Special Coordination Funds for promoting Science and Technology from the Science and Technology Agency of Japan, and by a Human Frontier Science Program grant to M. Kawato.

Address for reprint requests: M. Kawato, ATR Human Information Processing Research Laboratories, 2-2 Hikari-dai, Seika-cho, Soraku-gun, Kyoto 619-0288, Japan.

Received 14 April 1998; accepted in final form 7 January 1999.

\section{REFERENCES}

Abend, W., Bizzi, E., AND Morasso, P. Human arm trajectory formation. Brain 105: 331-348, 1982.

AKAZAWA, K. Modulation and adaptation of mechanical properties of mammalian skeletal muscle. In: Clinical Biomechanics and Related Research, edited by Y. Hirasawa, C. B. Sledge, and S.L.-Y. Woo. Tokyo: SpringerVerlag, 1994, p. 217-227.

AtKeson, C. G. AND Hollerbach, J. M. Kinematic features of unrestrained vertical arm movements. J. Neurosci. 5: 2318-2330, 1985.

BenNET, D. J. Torques generated at the human elbow joint in response to constant position errors imposed during voluntary movements. Exp. Brain Res. 95: 488-498, 1993.

Bennet, D. J., Hollerbach, J. M., Xu, Y., And Hunter, I. W. Time-varying stiffness of human elbow joint during cyclic voluntary movement. Exp. Brain Res. 88: 433-442, 1992.

BernsteIn, N. The Coordination and Regulation of Movements. Oxford, UK: Pergamon, 1967.

Bizzi, E., Accornero, N., Chapple, W., And Hogan, N. Posture control and trajectory formation during arm movement. J. Neurosci. 4: 2738-2744, 1984.

Conditt, M. A., Gandolfo, F., And Mussa-Ivaldi, F. A. The motor system does not learn the dynamics of the arm by rote memorization of past experience. J. Neurophysiol. 78: 554-560, 1997.

Dornay, M., Uno, Y., KaWATO, M., AND SuZUKI, R. Minimum muscle-tension change trajectories predicted by using a 17-muscle model of the monkey's arm. J. Mot. Behav. 28: 83-100, 1996.

Flanagan, J. R. AND RaO, A. K. Trajectory adaptation to a nonlinear visuomotor transformation: evidence of motion planning in visually perceived space. J. Neurophysiol. 74: 2174-2178, 1995.

FLASH, T. The control of hand equilibrium trajectories in multi-joint arm movements. Biol. Cybern. 57: 257-274, 1987.

FLASH, T. The organization of human arm trajectory control. In: Multiple Muscle Systems, edited by J. M. Winters and S.L.-Y. Woo. New York: Springer-Verlag, 1990, p. 282-301.

Flash, T. AND GuREvich, I. Arm stiffness and movement adaptation to external loads. Proc IEEE Eng. Med. Biol. Soc. 13: 885-886, 1991.

Flash, T. AND Hogan, N. The coordination of arm movements: an experimentally confirmed mathematical model. J. Neurosci. 5: 1688-1703, 1985.

Foley, J. M. Binocular distance perception. Psychol. Rev. 87: 411-434, 1980.

Fuchs, A. F., Scudder, C. A., AND KaneKo, C. R. Discharge patterns and recruitment order of identified motoneurons and internuclear neurons in the monkey abducens nucleus. J. Neurophysiol. 60: 1874-1895, 1988.

Georgopoulos, A. P., Kalaska, J. F., Caminiti, R., and Massey, J. T. On the relations between the direction of two-dimensional arm movements and cell discharge in primate motor cortex. J. Neurosci. 2: 1527-1537, 1982.

Georgopoulos, A. P., Schwartz, A. B., and Kettner, R. E. Neuronal population coding of movement direction. Science 233: 1416-1419, 1986.

Gomi, H. AND GotTLIEB, G. L. Joint torque and EMG pattern during multijoint arm movements in different inertial-viscosity force fields. In: 27th Annual 
Meeting of the Society for Neuroscience. New Orleans, LA: Soc. Neurosci., 1997, vol. 23, part 2, p. 2095.

Gomi, H. AND KaWato, M. Equilibrium-point control hypothesis examined by measured arm stiffness during multijoint movement. Science 272: 117-120, 1996.

Gomi, H. AND KaWATO, M. Human arm stiffness and equilibrium-point trajectory during multi-joint movement. Biol. Cybern. 76: 163-171, 1997.

Gomi, H. AND Osu, R. Task dependent viscoelasticity of human multijoint-arm and its spatial characteristics for interaction with environments. J. Neurosci. 18: 8965-8978, 1998.

Gomi, H., Shidara, M., Takemura, A., Inoue, Y., Kawano, K., and Kawato, M. Temporal firing patterns of Purkinje cells in the cerebellar ventral paraflocculus during ocular following responses in monkeys. I. Simple spikes. J. Neurophysiol. 80: 818-831, 1998.

Gribble, P. L., Ostry, D. J., Sanguineti, V., and Laboissiere, R. Are complex control signals required for human arm movement? J. Neurophysiol. 79: 1409-1424, 1998

HAGGARD, P. AND RichaRdSON, J. Spatial patterns in the control of human arm movement. J. Exp. Psychol. 22: 42-62, 1996.

HARRIS, C. M. AND WolPERT, D. M. Signal-dependent noise determines motor planning. Nature 394: 780-784, 1998.

Hollerbach, J. M. Planning of arm movement. In: Visual Cognition and Action, edited by D. N. Osherson, S. M. Kosslyn, and J. M. Hollerbach. Cambridge, MA: MIT Press, 1990, p. 183-211.

ImAMizU, H., UNO, Y., AND KaWATO, M. Internal representations of the motor apparatus: implications from generalization in visuomotor learning. J. Exp. Psychol. 21: 1174-1198, 1995.

INDOW, T. AND Watanabe, T. Alleys on an extensive apparent frontoparallel plane: a second experiment. Perception 17: 647-666, 1988.

Katayama, M. And Kawato, M. Virtual trajectory and stiffness ellipse during multijoint arm movement predicted by neural inverse models. Biol. Cybern. 69: 353-362, 1993

KawATO, M. Optimization and learning in neural networks for formation and control of coordinated movement. In: Attention and Performance XIV, edited by D. E. Meyer D. E. and S. Kornblum. Cambridge, MA: MIT Press, 1992, p. 821-849.

KaWATO, M. Trajectory formation in arm movements: minimization principles and procedures. In: Advances in Motor Learning and Control, edited by H. N. Zelaznik. Champaign, IL: Human Kinetics Publishers, 1996, p. 225-259.

KelLeR, E. L. Accommodative vergence in the alert monkey. Motor unit analysis. Vision Res. 13: 1565-1575, 1973.

Kelso, J.A.S., Southard, D. L., AND Goodman, D. On the nature of human interlimb coordination. Science 203: 1029-1031, 1979.

KoIKE, Y. AND KAWATO, M. Estimation of dynamic joint torques and trajectory formation from surface electromyography signals using a neural network model. Biol. Cybern. 73: 291-300, 1995.

LACKNER, J. R. AND DizIO, P. Rapid adaptation to coriolis force perturbations of arm trajectory. J. Neurophysiol. 72: 299-313, 1994.

Lacquaniti, F., Guigon, E., Bianchi, L., Ferraina, S., and Caminiti, R. Representing spatial information for limb movement: role of area 5 in the monkey. Cereb. Cortex 5: 391-409, 1995.

Morasso, P. Spatial control of arm movements. Exp. Brain Res. 42: 223-227, 1981.
NeLSON, W. L. Physical principles for economies of skilled movements. Biol. Cybern. 46: 135-147, 1983.

OKADOME, T. AND HoNDA, M. Trajectory formation in sequential arm movements. In: IEEE International Conference on Systems Man and Cybernetics. Chicago, IL: IEEE, 1992, vol. 1, p. 471-478.

Osu, R., Uno, Y., Koike, Y., and Kawato, M. Possible explanations of trajectory curvature in multijoint arm movements. J. Exp. Psychol. Hum. Percept. and Perform. 23: 890-913, 1997.

ÖZKAYA, N. AND NoRBIN, M. Fundamentals of Biomechanics: Equilibrium, Motion, and Deformation. New York: Van Nostrand Reinhold, 1991.

Rosenbaum, D. A., Loukopoulos, L. D., Meulenbroek, R. G., Vaughan, J., AND ENGELBRECHT, S. E. Planning reaches by evaluating stored postures. Psychol. Rev. 102: 28-67, 1995.

SALTZMAn, E. L. AND Kelso, J.A.S. Skilled actions: a task-dynamics approach. Psychol. Rev. 94: 84-106, 1987.

SCOTT, S. H. AND KalasKa, J. F. Changes in motor cortex activity during reaching movements with similar hand paths but different arm postures. J. Neurophysiol. 73: 2563-2567, 1995.

ScotT, S. H. AND KalaSKa, J. F. Reaching movements with similar hand paths but different arm orientations. I. Activity of individual cells in motor cortex. J. Neurophysiol. 77: 826-852, 1997.

Sergio, L. E. AND Kalaska, J. F. Changes in the temporal pattern of primary motor cortex activity in a directional isometric force versus limb movement task. J. Neurophysiol. 80: 1577-1583, 1998.

ShadmeHR, R. AND Mussa-Ivaldi, F. A. Adaptive representation of dynamics during learning of a motor task. J. Neurosci. 14: 3208-3224, 1994

Shidara, M., Kawano, K., Gomi, H., and Kawato, M. Inverse-dynamics model eye movement control by Purkinje cells in the cerebellum. Nature 365: 50-52, 1993.

SoEchting, J. F. and Lacquaniti, F. Invariant characteristics of a pointing movement in man. J. Neurosci. 1: 710-720, 1981.

Uno, Y., Imamizu, H., Gomi, H., and Kawato, M. Space where arm trajectory is planned: evidence from experiments in altered dynamics. In: Fourth Annual Meeting of IBRO World Congress of Neuroscience. Sapporo, Japan: Japan Neuroscience Society, 1995, D6.33, p. 343.

Uno, Y. and Kawato, M. The trajectories of human arm movements depend on movement duration. In: Eleventh Symposium on Biological and Physiological Engineering (in Japanese). Osaka, Japan: SICE, 1996, p. 329-332.

Uno, Y., Kawato, M., AND Suzuki, R. Formation and control of optimal trajectory in human multijoint arm movement: minimum torque-change model. Biol. Cybern. 61: 89-101, 1989a.

Uno, Y., Suzuki, Y., and Kawato, M. Minimum muscle-tension-change model which reproduces human arm movement. In: Fourth Symposium on Biological and Physiological Engineering (in Japanese). Tokyo, Japan: SICE, 1989b, p. 299-302.

Wolpert, D. M., Ghahramani, Z., AND Jordan, M. I. Perceptual distortion contributes to the curvature of human reaching movements. Exp. Brain Res. 98: 153-156, 1994

Wolpert, D. M., Ghahramani, Z., AND Jordan, M. I. Are arm trajectories planned in kinematic or dynamic coordinates? An adaptation study. Exp. Brain Res. 103: 460-470, 1995. 